\title{
Local transport of passive scalar released from a point source in a turbulent boundary layer
}

\author{
K. M. Talluru ${ }^{1} \dagger$, J. Philip ${ }^{2}$ and K. A. Chauhan ${ }^{1}$ \\ ${ }^{1}$ School of Civil Engineering, The University of Sydney, NSW 2006, Australia \\ ${ }^{2}$ Department of Mechanical Engineering, The University of Melbourne, VIC 3010, Australia \\ (Received ?; revised ?; accepted ?. - To be entered by editorial office)
}

Simultaneous measurements of streamwise velocity $(\tilde{U})$ and concentration $(\tilde{C})$ for a horizontal plume released at eight different vertical locations within a turbulent boundary layer are discussed in this paper. These are supplemented by limited simultaneous three-component velocity and concentration measurements. Results of integral time-scale $\left(\tau_{c}\right)$ of concentration fluctuations across the width of the plume are presented here for the first time. It is found that $\tau_{c}$ has two distinct peaks; one closer to the plume centreline and the other at a vertical distance of plume half-width above the centreline. The time-averaged streamwise concentration flux is found to be positive and negative respectively, below and above the plume centreline. This behaviour is a resultant of wall-normal velocity fluctuations $(w)$ and Reynolds shear stress $(\overline{u w})$. Confirmation of these observations is found in the results of joint-probability density functions of $u$ (streamwise velocity fluctuations) and $\tilde{C}$ as well as that of $w$ and $\tilde{C}$. Results of cross-correlation coefficient show that high- and low- momentum regions have a distinctive role in the transport of passive scalar. Above the plume centreline, lowspeed structures have a lead over the meandering plume, while high-momentum regions are seen to lag behind the plume below its centreline. Further examination of the phase relationship between time-varying $u$ and $c$ (concentration fluctuations) via cross-spectrum analysis is consistent with this observation. Based on these observations, a phenomenological model is presented for the relative arrangement of a passive scalar plume with respect to large-scale velocity structures in the flow.

Key words: turbulent boundary layer, integral time-scales, velocity-concentration correlations, scalar transport

\section{Introduction}

Scalar contaminants such as toxic gases and pollutants are often inadvertently introduced into a background flow (e.g. in the atmosphere boundary layer) approximately at a point, or at source sizes that are significantly smaller compared to the domain of bulk flow. Dispersion of a passive scalar released from a point source in a turbulent boundary layer encompasses many features of the practical atmospheric flows, and is the focus of this study. The transport of scalar in a turbulent flow depends on both the instantaneous as well as the time-averaged properties of the scalar and the flow itself. This is clearly evident in the Reynolds averaged mean scalar and scalar-variance equations,

$$
\begin{aligned}
U_{i} \frac{\partial C}{\partial x_{i}} & =-\frac{\partial \overline{u_{i} c}}{\partial x_{i}}+D\left(\nabla^{2} C\right), \quad \text { and } \\
U_{i} \frac{\partial \overline{c c}}{\partial x_{i}} & =-\frac{\partial \overline{u_{i} c c}}{\partial x_{i}}+D\left(\nabla^{2} \overline{c c}\right)-2 \overline{u_{i} c} \frac{\partial C}{\partial x_{i}}-\chi_{c},
\end{aligned}
$$

respectively, that show the inherent coupling between the velocity $(\tilde{\mathbf{U}}=\tilde{U} \hat{i}+\tilde{V} \hat{j}+\tilde{W} \hat{k})$ and the scalar fields. Here, instantaneous streamwise velocity and concentration are denoted as $\tilde{U}_{i}=$

$\dagger$ Email address for correspondence: murali.talluru@sydney.edu.au. 
$U_{i}+u_{i}$ and $\tilde{C}=C+c$, respectively, $D$ represents the coefficient of molecular diffusion and $\chi_{c}=2 D \overline{\left(\frac{\partial c}{\partial x_{i}}\right)\left(\frac{\partial c}{\partial x_{i}}\right)}$ is the rate of dissipation of scalar variance. Note that upper case letters or the over-line represents the time-averaged (mean) quantities, whereas the corresponding lower case letters denote the fluctuating quantities defined about the mean. Thus an understanding of instantaneous processes within a plume is essential for establishing a description of the physics that controls the time-averaged properties.

Several past studies have examined scalar dispersion and also developed statistical models for its prediction in both convective boundary layers (Townsend 1958; Arya 1975; Willis \& Deardorff 1978, among others) and rough-wall turbulent boundary layers (Poreh \& Cermak 1964; Fackrell \& Robins 1982; Sawford et al. 1985; Yee et al. 1993; Nironi et al. 2015). However, there are no known studies of scalar dispersion in canonical smooth-wall turbulent boundary layers. The emphasis on roughwall boundary layers is understandable since atmospheric flows are characteristically developing over some form of roughness. One of the aims of current study is to fill this gap of experiments over smooth walls and to associate the substantial knowledge of turbulence structure in wall-bounded flows with dispersion. Thereby, we also aim to understand the influence of coherent motions in boundary layer flows on scalar transport, as such motions also play an important role in the transport of momentum and vorticity across the boundary layer (Marusic et al. 2010; Smits et al. 2011; Klewicki 2013).

For a plume released from a point source, Fackrell \& Robins (1982) and Nironi et al. (2015), both using Flame Ionisation Detection (FID) technique for measuring instantaneous concentration, have shown that the mean and variance of concentration fluctuations in a rough-wall turbulent boundary layer exhibit either reflected Gaussian or Gaussian behaviour depending on the source height. Fackrell \& Robins (1982) found that a ground level plume tends to disperse in a Gaussian distribution (to a close approximation) while an elevated plume exhibits a reflected-Gaussian behaviour, which has been further substantiated by Nironi et al. (2015). Both these studies considered a ground level and a single elevated source and thus could not clarify the influence of source height on plume dispersion. To this end, the current study reports experimental data for different source heights in the near wall region, log-layer, fully-turbulent outer region and intermittent region of a boundary layer.

Another parameter relevant to plume dispersion is the source size (Chatwin \& Sullivan 1979; Durbin 1980). The influence of source size can be examined by considering the Gaussian plume model put forth by Gifford (1959). He suggested that the concentration fluctuations are governed by two distinct phenomena - meandering of the instantaneous plume and relative dispersion of the plume particles. In general, the meandering is due to the presence of wide range of length-scales in the flow relative to the source size. Using the results of Hay \& Pasquill (1959) and Smith \& Hay (1961) on relevant scales for plume dispersion, Fackrell (1980) showed that meandering is the major source of concentration fluctuations in a developing plume. Experimental studies (Fackrell \& Robins 1982; Nironi et al. 2015) have concluded that the source size does not influence a ground level plume since the turbulent length-scales near the wall are typically smaller or comparable to the source size. On the other hand, the source diameter influences the structure of an elevated plume as the turbulent eddies are nominally larger than the source diameter. For an elevated source, the results from (Fackrell \& Robins 1982; Nironi et al. 2015) conclusively show that the variation of source size does not alter the mean concentration field but affects the concentration fluctuations, which is the case in the current study, i.e. $s_{d} / \delta \approx 0.0025$, where $s_{d}$ is the source diameter.

The seminal study of Taylor (1921) shows that the spread of a scalar depends on the Lagrangian integral time-scales in the flow, and since then there have been efforts to estimate them using the the Eulerian integral time-scales $\left(\tau_{c}\right)$, which are experimentally more easily tractable. Eulerian autocorrelation functions and corresponding time-scales are important quantities in their own right, and a few studies, such as, Antonia et al. (1977), Tavoularis \& Corrsin (1981) and Krishnamoorthy \& Antonia (1987) have employed temperature as a passive scalar using cold-wires (with constant current anemom- 
etry) to measure temperature fluctuations with high temporal resolution. Although temperature is a suitable passive scalar, it is challenging to implement its release from a source in close vicinity of a surface due to conduction at physical boundaries, i.e. the boundary condition of zero flux at the wall, $\mathrm{d} \tilde{C} / \mathrm{d} z=0$ at $z=0$ cannot be ensured ( $z$ is the distance from the wall). On the other hand, the use of gas as a passive scalar does not suffer this limitation due to non-permeability of the wall. However, measuring gas concentration at high frequency is difficult and currently available instrumentation such as flame-ionisation and photo-ionisation detectors have typical response of up to $400 \mathrm{~Hz}$. Further, these sensors are prone to drift in time due to humidity, variation in temperature and changes in background concentration of the flow. To minimize the effect of sensor drift on statistics, we have successfully implemented systematic calibration procedures (Talluru et al. 2017a) for photo-ionisation detectors (that could also be implemented for flame-ionisation detectors), and consequently an accurate computation of $\tau_{c}$ is possible, results of which will be presented here. Fackrell \& Robins (1982) is the only study, to our knowledge, that reported the statistics of $\tau_{c}$ for a ground level and elevated plumes and observed that $\tau_{c}$ is higher near the wall. Further, with only few measurement points across the plume, they found that there is little variation of $\tau_{c}$ vertically for an elevated source. Here, we aim to document the behaviour of $\tau_{c}$ across the plume from a point source released at various heights, and to understand its variation.

In the past few decades, numerous studies have also reported the presence of coherent structures in various turbulent flows and more recently, these structures have been identified to contribute to the generation of Reynolds stresses, which play a dominant role in the transport of momentum. There is evidence that coherent motions are responsible for heat and momentum fluxes in the atmospheric surface layer (Katul et al. 1997; Finnigan et al. 2009; Li \& Bou-Zeid 2011; Huang et al. 2013). Further, from the presence of mean and fluctuation velocity in equations $(1.1 a)$ and $(1.1 b)$, it follows that coherent structures would also be significant for scalar transport. However, the exact nature of scalar transport by coherent features is not well-understood possibly due to experimental complexity in obtaining simultaneous measurements of velocity and concentration with good spatial and temporal resolution. Recently Vanderwel \& Tavoularis (2016) observed that a scalar dye segregated preferentially within vortex cores and the boundaries of uniform momentum zones (see Meinhart \& Adrian 1995 for a description of uniform momentum zones) in a uniformly sheared flow. Vanderwel \& Tavoularis (2016) also utilized conditional analysis to show that strong scalar flux events are well-correlated with Reynolds shear stress events. Thus it is imperative to understand transport of scalar by known coherent features such as hair-pin packets (e.g. Adrian et al. 2000), meandering super-structures (e.g. Hutchins \& Marusic 2007a), vortical motions (e.g. Klewicki et al. 1994; Herpin et al. 2013) and uniform momentum zones (e.g. de Silva et al. 2017). Here, analysis of cross-correlation and cross-spectra of instantaneous velocity and concentration will provide insights into the organisation of scalar and velocity structures.

The paper is outlined as follows: $\S 2$ provides details of the experimental set-up and measurement devices. In $\S 3$, we first present results for mean and variance of concentration as the source location is varied. This is followed by the evaluation of $\tau_{c}$ and $\tau_{u}$, and spectral decomposition of scalar and velocity signals. The influence of velocity on scalar is discussed in $\S 4$, along-with concentration flux $(\overline{u c})$, cross-correlation, and cross-spectral density. Further results from joint-probability density function (j-pdf) are also included in $\S 4$, and our phenomenological plume model to explain results and conclusions follow in $\S 5$.

\section{Details of experiments}

Experiments are conducted in the Boundary Layer Wind Tunnel (BLWT) located at the University of Sydney. A schematic of the experimental set-up is shown in figure 1, where simultaneous measurements of velocity and concentration are made using a single hotwire and a photo-ionisation detector (PID) respectively. In the present study, the free-stream velocity is set to $10 \mathrm{~m} / \mathrm{s}$, which re- 


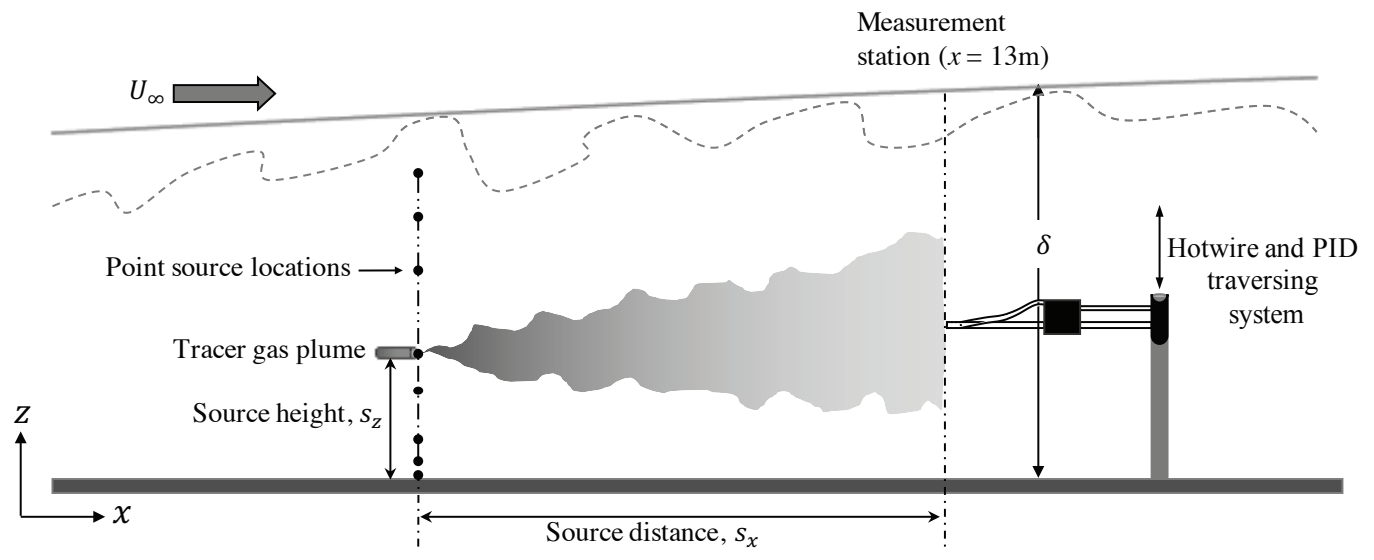

FIGURE 1. Schematic of experimental set-up showing the arrangement of hotwire and PID and different parameters of the point source.

sulted in a boundary layer thickness, $\delta \approx 0.31 \mathrm{~m}$ and friction velocity, $U_{\tau} \approx 0.37 \mathrm{~m} / \mathrm{s}$ (obtained by fitting mean velocity data to the composite velocity fit given by Chauhan et al. 2009 using log-law constants $\kappa=0.384$ and $A=4.17$ ) at the measurement location $x=13 \mathrm{~m}$ downstream of the trip. The friction Reynolds number, $R e_{\tau} \equiv \delta U_{\tau} / \nu=\delta^{+} \approx 7850$ ( $\nu$ is the kinematic viscosity of air) at the measurement location and other parameters of the boundary layer are listed in table 1 . Note that normalisation using wall units is denoted by a ' + ' superscript in this paper, e.g. $l^{+}=l /\left(\nu / U_{\tau}\right)$ and $U^{+}=U / U_{\tau}$, where $l$ is the hotwire sensor length and $U$ is the mean streamwise velocity. The mean velocity and turbulence intensity profiles were previously documented in (Talluru et al. 2017a), and at the measurement location closely resemble a zero-pressure-gradient turbulent boundary layer. Full details of the wind tunnel facility and the calibration procedures for PID sensors are given in Talluru et al. (2017a). In the case of hotwire measurements, procedures outlined in Talluru et al. (2014) are employed to correct for any voltage drift during the measurements. Throughout this paper, $x, y$ and $z$ refer to the streamwise, transverse and vertical directions while $u, v$ and $w$ denote the corresponding fluctuating velocity components.

A point source tracer gas (a mixture of $1.5 \%$ Iso-Butylene and 98.5\% Nitrogen) with an initial concentration $\left(C_{0}\right)$ of $15000 \mathrm{ppm}$ is released via a discharge tube at eight different heights $\left(s_{z}\right.$, as listed in table 1) above the tunnel floor, and measurements are made at a fixed distance $s_{x} / \delta=1$ from the source (as illustrated in figure 1). The source heights were chosen to observe variation in local spread of scalar plume due to varying properties of local turbulence across the boundary layer. The location $s_{z} / \delta=32 / \delta^{+}$, which is physically $1.26 \mathrm{~mm}$ from the ground (compared to a $\delta=310 \mathrm{~mm}$ ) is the closest we can release the gas and is called the ground source; $s_{z} / \delta=350 / \delta^{+}$ and $s_{z} / \delta=0.1$ are within the inertial or log-region of the boundary layer (note that the start of the log-region is at $\approx 3.6 \sqrt{\delta^{+}}$as shown recently by Morrill-Winter et al. 2017, which in our case is 319 viscous units); $s_{z} / \delta=0.25$ is in the fully-turbulent outer region; $s_{z} / \delta=0.5$ is in the mostly turbulent outer region (note that external intermittency begins near $z / \delta=0.33$, Chauhan et al., $2014 b), s_{z} / \delta=0.67$ corresponds to a location where the flow is non-turbulent $50 \%$ of time, and $s_{z}=\delta_{99}$ corresponds to location near the outer edge where the boundary layer is mostly nonturbulent.

The tracer gas is released iso-kinetically into the flow by matching the source velocity $\left(U_{s}\right)$ with the local mean velocity at $z=s_{z}$, i.e. $U_{s}=\left.U\right|_{\left(z=s_{z}\right)}$. It is important to note here that there is a time lag between the measured instantaneous velocity and concentration signals due to delay in the response of PID sensor compared to hotwire. The time lag is the time required for the gas sample to travel from the sensor tip to the detection chamber of PID. Diagnostic tests conducted by 

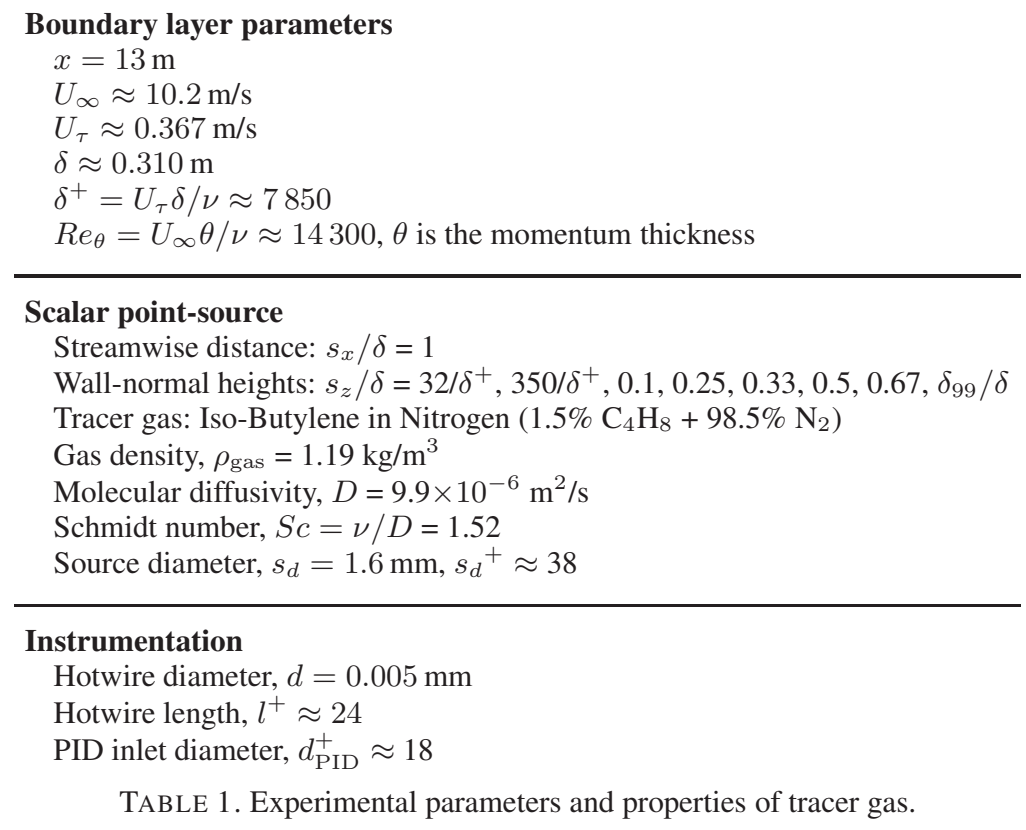

Talluru et al. (2017b) found that PID signal has an average time lag of 10 milli-seconds with respect to hotwire signal, and hence, all the concentration signals are accordingly shifted in time for analysis. The effect of uncertainty in time-lag between velocity and concentration signals on time-averaged streamwise flux $(\overline{u c})$ was studied by systematically varying the time-lag while post-processing the data. It is found that an uncertainty of 2 milli-seconds resulted in $8 \%$ error in $\overline{u c}$. Hence, extra care must be exercised to determine the time-lag accurately as done in the current study.

In our previous study (Talluru et al. 2017a), we conducted several experiments to quantify the influence of discharge tube and the PID. It was found that the operation of PID has a minimal influence on the mean $( \pm 1 \%)$ and turbulence $( \pm 2 \%)$ statistics of velocity. On the other hand, the presence of the tracer gas discharge tube has resulted in a noticeable influence $( \pm 5 \%)$ on turbulence intensity of the flow, particularly in the case of ground and low level sources.

A second set of experiments are conducted using a four-hole pressure 'Cobra' probe capable of measuring three components of velocity, simultaneously sampled with PID (for scalar). These measurements, however, are limited to only three locations, i.e. at the plume centreline $\left(s_{z}\right)$ and at two locations on either side of plume centreline, $s_{z} \pm \delta_{\sigma}$. Here, $\delta_{\sigma}$ represents the plume half-width as defined later in section 3. The Cobra probe is available from Turbulent Flow Instrumentation, Inc. Australia, that provides instantaneous three-component velocity and local static pressure measurements. It has a frequency response of more than $2 \mathrm{kHz}$. A custom built mount is used to hold the Cobra probe in close proximity to the PID, similar to the set-up used for simultaneous hotwire-PID measurements. The time lag between Cobra probe and PID is also found to be approximately 10 milli-seconds as observed in hotwire-PID measurements, confirming that the time delay is essentially due to the length of flexible tube that connects the PID inlet to its ionisation chamber.

\section{Independent statistics of concentration and velocity}

Although velocity and concentration measurements are performed simultaneously, statistics from velocity and concentration signal are independently assessed in this section. 

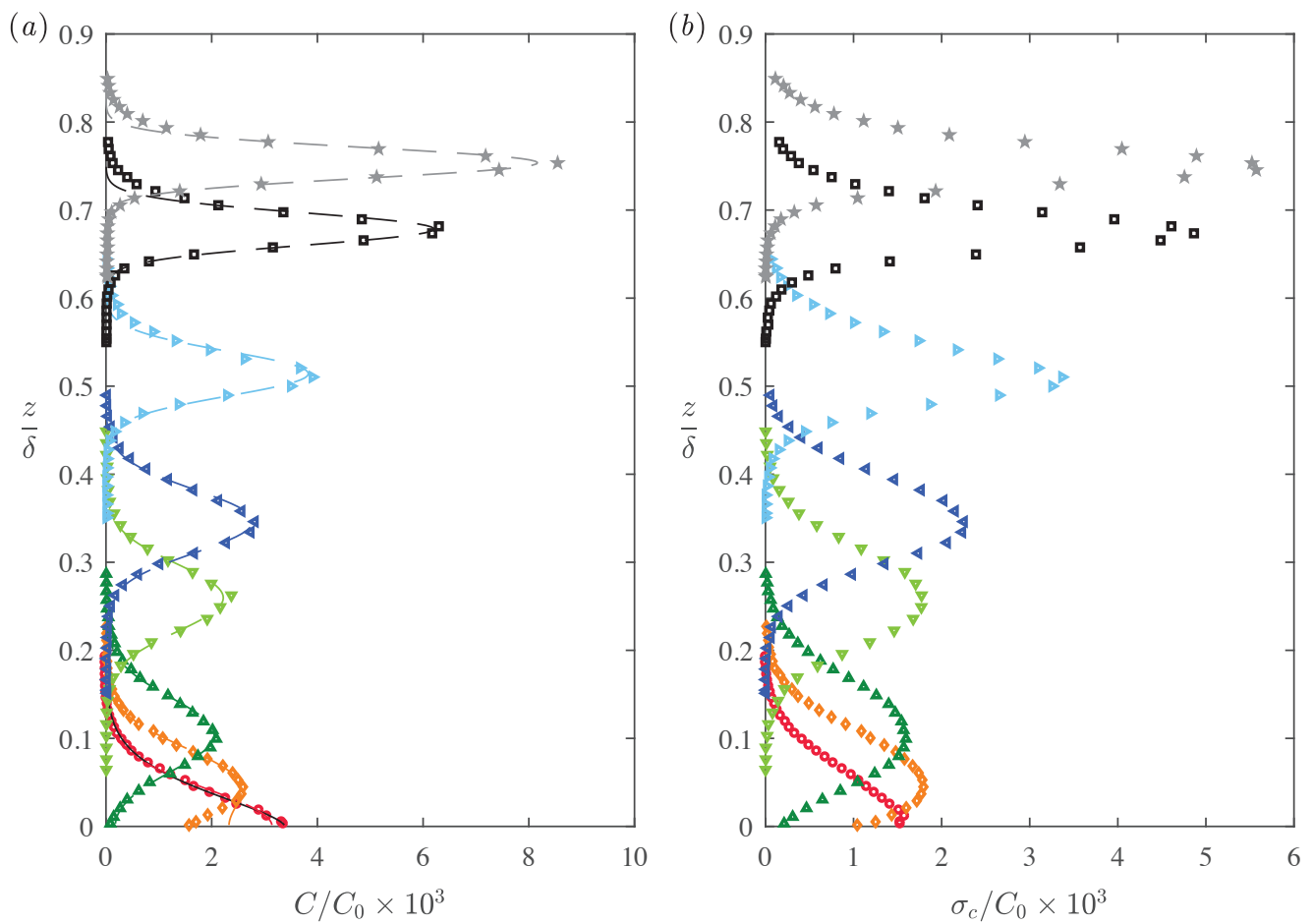

FIGURE 2. Profiles of normalised $(a)$ mean and $(b)$ r.m.s of concentration as a function of $z / \delta$ for all source heights. Note that initial concentration of tracer gas $\left(C_{0}=15,000 \mathrm{ppm}\right)$ is used for normalisation. Symbols: $s_{z} / \delta=32 / \delta^{+}(\bigcirc), 350 / \delta^{+}(\diamond), 0.1(\triangle), 0.25(\nabla), 0.33(\triangleleft), 0.5(\triangleright), 0.67(\square), \delta_{99} / \delta(*)$. Dashed lines in the mean concentration profiles represent the reflected Gaussian model while the black solid line (only for $s_{z} / \delta=32 / \delta^{+}$) shows the exponential fit.

\subsection{Mean and r.m.s profiles of concentration}

Results of mean, $C$ and r.m.s., $\sigma_{c}$ profiles of concentration for different source heights $s_{z}$ are plotted in figures $2(a)$ and $2(b)$, respectively. Note that the profiles are represented in the normalised form using the initial concentration of tracer gas, $C_{0}$. The wall-normal distribution of $C$ can be modelled from the streamwise component of equation (1.1a) by assuming an approximation for the vertical flux, $\overline{w c}$ using the Reynolds' analogy (of gradient diffusion theory) and neglecting viscous diffusion (e.g. Csanady 1973). One such model for elevated source, that is widely adopted, is the reflected Gaussian distribution for $C$ (obtained as solution of the diffusion equation with constant diffusivity and zero flux at the wall), and is given as (e.g. Csanady 1973; Fackrell \& Robins 1982):

$$
C(z)=A\left[\exp \left\{-B\left(\frac{z-s_{z}}{\delta_{C}}\right)^{2}\right\}+\exp \left\{-B\left(\frac{z+s_{z}}{\delta_{C}}\right)^{2}\right\}\right],
$$

where, $A$ and $B$ are parameters obtained by fitting the data to the equation, and $\delta_{C}$ is the vertical plume half-width, which is defined as the vertical distance (with respect to the source height) where local concentration is equal to half of the maximum mean concentration. The fit is shown in figure $2(a)$ by dashed lines, whereas $\delta_{C}$ for various source locations are plotted in figure 3 . This fit represents the elevated sources well, except perhaps for the case of $s_{z} / \delta=350 / \delta^{+}$close to the ground, and definitely not the ground level source.

For plumes emanating from a ground level source and which exhibit self-preserving form of the vertical profile of mean concentration, the use gradient diffusion theory suggests a solution (Csanady 


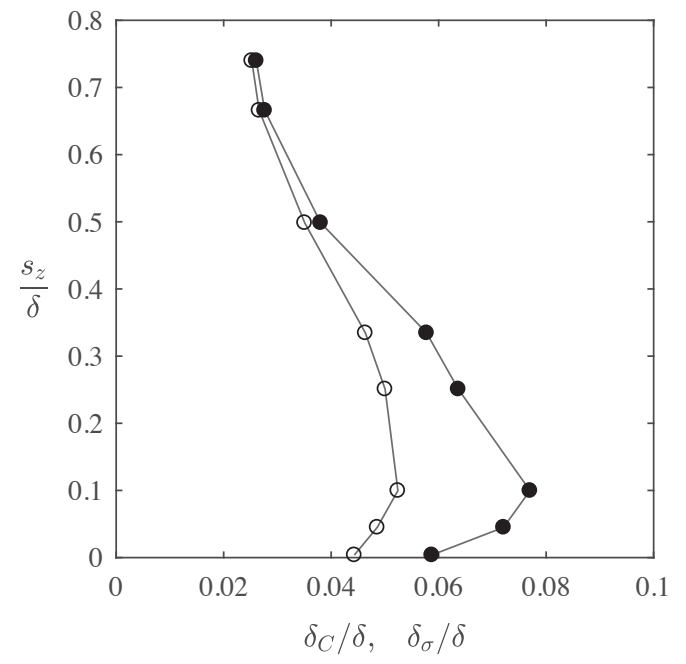

FIGURE 3. Plume half-widths obtained from mean $\left(\delta_{C}\right.$, open symbols) and r.m.s ( $\delta_{\sigma}$, filled symbols) profiles of concentration for different source heights.

1973; Robins 1978) as,

$$
C(z)=C_{0}\left[\exp \left\{-b\left(\frac{z}{\delta_{C}}\right)^{\beta}\right\}\right],
$$

where, $b=\ln (2)$, and $\beta$ is a parameter that depends on surface roughness (Csanady 1973; Hunt \& Weber 1979). For instance, Fackrell \& Robins (1982) obtained a value of 1.5 for $\beta$ in a rough wall boundary layer while Robins (1978) reported a slightly higher value of $\beta=1.7$ in a rougher boundary layer. For our case, the ground level source $\left(s_{z} / \delta=32 / \delta^{+} \approx 0.0041\right)$ presented in figure $2(a)$ is well described by equation (3.2) with $\beta=1.5$, which is close to the value reported by Fackrell \& Robins (1982), and is indicated by a solid (black) line. For several engineering applications, the turbulent boundary layer mean velocity profile can be approximated with a power law profile, $U=U_{\infty}(z / \delta)^{\alpha}$, where $\alpha$ is the power exponent, usually taken to be $1 / 7$. In equation (3.2), $\beta$ can be shown to be equal to $(1+2 \alpha)$ (Csanady 1973), which is not unreasonable.

We also present the wall-normal profiles of r.m.s, $\sigma_{c}$ of concentration for different source locations in figure $2(b)$, and the corresponding half-width $\delta_{\sigma}$ (determined from the location where $\sigma_{c}$ is half its peak centreline value) is shown in figure 3 . The plume half-widths, $\delta_{C}$ and $\delta_{\sigma}$ characterise the spatial extent where significant concentration events can be expected, albeit intermittently. In figure 3 , it is observed that, both $\delta_{C}$ and $\delta_{\sigma}$ increase up to $s_{z} / \delta=0.1$, beyond which they decrease monotonically. Further, $\delta_{\sigma}$ is found to be larger than $\delta_{C}$ for source heights $s_{z} / \delta \leq 0.5$, and there is negligible difference between the two at higher locations. In fact, we see that the width $\delta_{\sigma}$ corresponding to $\sigma_{C}$ is noticeably larger than $\delta_{C}$ in the log-region, where high turbulence intensity and strong shear exists. Similar observations of $\delta_{\sigma}$ greater than $\delta_{C}$ are made by Vanderwel \& Tavoularis (2014) in a uniformly sheared flow. This is also consistent with the well-accepted meandering plume model of Gifford (1959), where an 'instantaneous' Gaussian plume, the width of which fluctuates randomly across the transverse direction, gives rise to an increased fluctuation width compared to the mean (see also, Weil 2012).

In the present study, we have not investigated a model to fit the r.m.s. profiles, as such a model would require validation with varying streamwise source distance $s_{x}$, which is beyond the scope of this paper. The aim here is to understand the role of velocity structures in scalar transport, and as such, the data of $C$ and $\sigma_{c}$ presented here establish confidence in the measurements. Also note that, henceforth, the plume half-width refers to the $\delta_{\sigma}$ based on $\sigma_{c}$ profiles. 
(a)

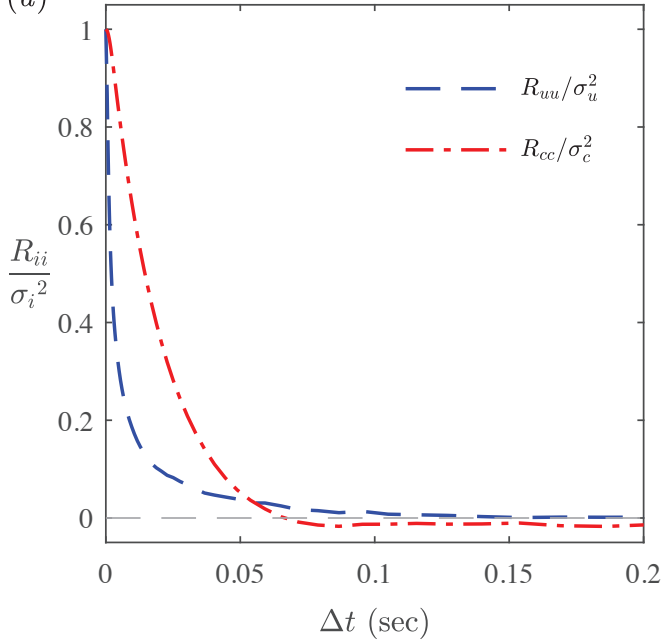

(b)

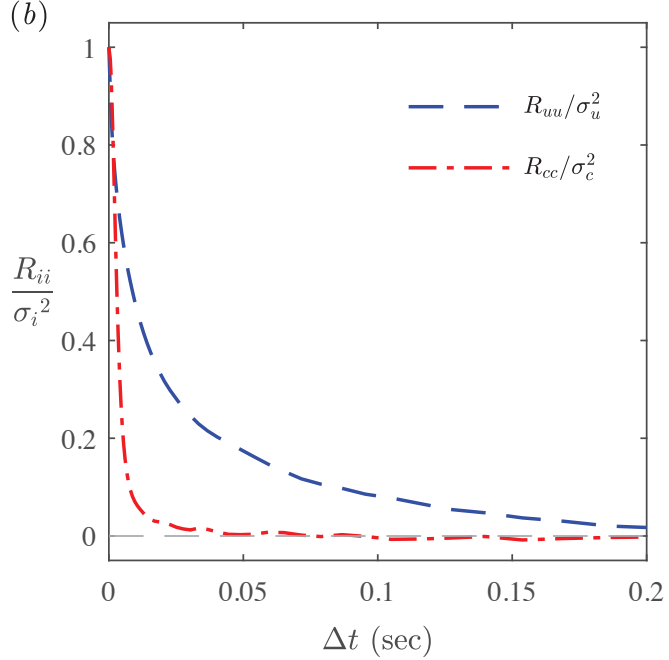

FIgURE 4. Comparison of $R_{i i} / \sigma_{i}{ }^{2},(i=u, c)$ at the centreline of the plume for $(a) s_{z} / \delta=32 / \delta^{+}$, the ground-level source, where $\tau_{u}(0.008 \mathrm{sec})<\tau_{c}(0.017 \mathrm{sec})$, and $(b) s_{z} / \delta=0.1$, the log-region source, where $\tau_{u}(0.028 \mathrm{sec})>\tau_{c}(0.004 \mathrm{sec})$. Note that $\tau_{c}$ is calculated by integrating $R_{c c} / \sigma_{c}{ }^{2}$ up to its first zero-crossing, while $\tau_{u}$ is obtained by integrating $R_{u u} / \sigma_{u}{ }^{2}$ in the interval $0 \leq \Delta t \leq 1.2 \mathrm{sec}$ that is equivalent to a streamwise length of $40 \delta$.

\subsection{Auto-correlation and integral time-scales}

The auto-correlation function $R_{i i}(\mathbf{x}, \Delta t)$ is defined as,

$$
R_{i i}(\mathbf{x}, \Delta t)=\overline{i(\mathbf{x}, t) i(\mathbf{x}, t+\Delta t)}, \quad i=u, c .
$$

Note that the auto-correlation function will be different for varying source heights $s_{z}$. For wallnormal profile measurements considered here, the integral time-scales of velocity, $\tau_{u}$ and concentration, $\tau_{c}$ are calculated from the auto-correlation function as,

$$
\tau_{i}(z)=\int_{0}^{\infty} \frac{R_{i i}\left(z, t^{\prime}\right)}{\left[\sigma_{i}(z)\right]^{2}} \mathrm{~d} t^{\prime},
$$

with $\sigma_{i}{ }^{2}$ as the variance of the fluctuating quantity.

The integral time-scales $\tau_{u}$ and $\tau_{c}$ indicate the average duration of a coherent velocity structure or a scalar/concentration region passing through measurement location. The integral time-scale for concentration, $\tau_{c}$ is relevant to estimates of 'exposure times', a measure of time-duration over which concentration levels exceed a certain threshold. It is found that $\tau_{c}$ is higher than $\tau_{u}$ for a ground level source as inferred from figure $4(a)$ showing $R_{c c}$ and $R_{u u}$ for $s_{z} / \delta=32 / \delta^{+}$. This may be attributed to two factors. First, intense small-scale activity of velocity fluctuations in the near-wall region leads to a faster decline of auto-correlation of $u$ in time, resulting in a smaller magnitude of $\tau_{u}$. Second, concentration tends to persist for longer time near the wall since the streamwise mean velocity is relatively low, and combined with the fact that concentration flux is zero at the wall, longer correlations and higher integral time-scales are measured. On the other hand, for all elevated sources, $\tau_{c}$ is typically smaller than $\tau_{u}$, as illustrated in figure $4(b)$ for source in the logarithmic region, with $R_{u u}$ and $R_{c c}$ again measured on the centre line of the plume. This in-turn implies that the length-scales associated with concentration are smaller than the length-scale of turbulent motions. This is consistent with the arguments put forth by Tennekes \& Lumley (1972) (also see Duplat \& Villermaux 2008), i.e., the turbulence will mix scalar at the smallest scales of velocity, resulting in a short correlation time, whereas velocity correlation times (at least in shear flows) are dominated by large coherent structures leading to a longer time-scales compared to the scalar. 

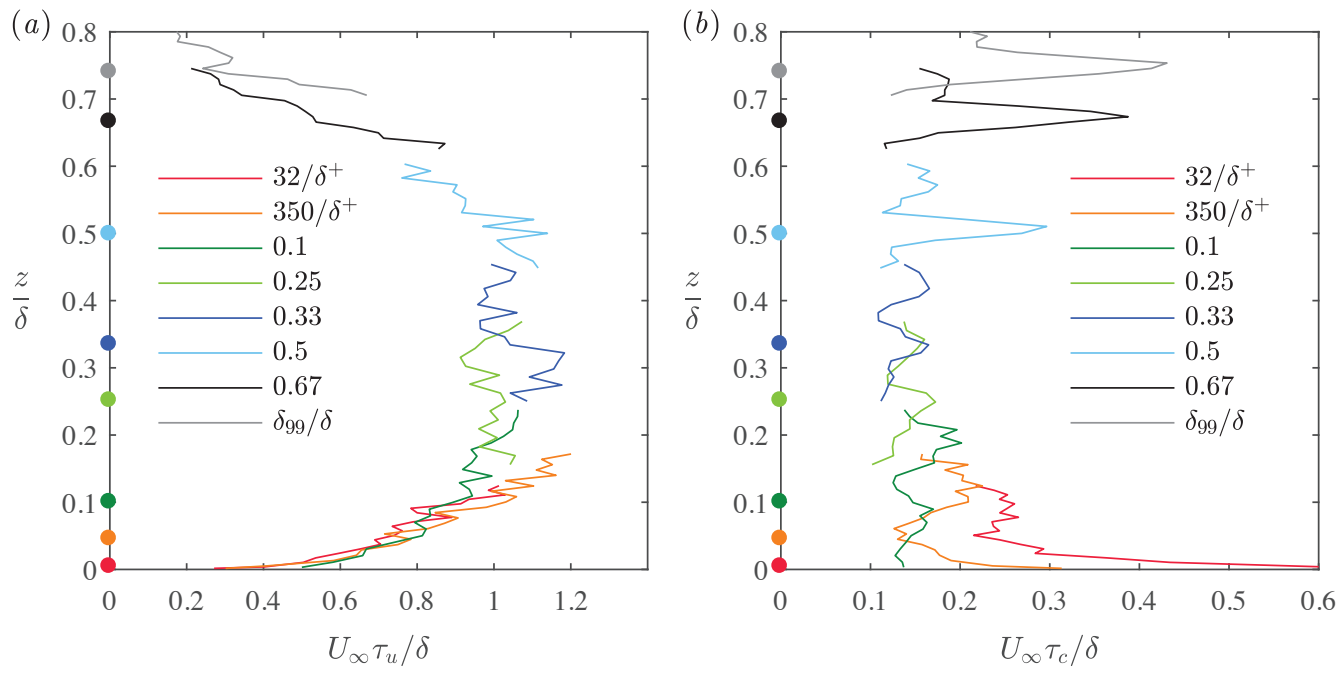

FIGURE 5. Comparison of integral time-scales of $(a)$ velocity and $(b)$ concentration fluctuations for different source heights indicated by colored dots on the ordinate axis.

As an aside, we note that in the Eulerian frame of reference, the integral length-scale for velocity fluctuations in a fully-developed turbulent flow can be computed from the integral time-scale by invoking Taylor's frozen turbulence hypothesis and assuming local mean velocity as the convection velocity. This has been shown to be approximately valid (Dennis \& Nickels 2008; Del Álamo \& Jiménez 2009) for velocity structures in wall-bounded flows. This hypothesis, however, is not applicable to a scalar concentration field that is rapidly changing downstream of a point source. Hence, the discussion here is limited to the integral time (rather than length) scales of $u$ and $c$ that are unambiguously calculated from single-point measurements.

Figures $5(a)$ and $5(b)$ show the integral time-scales of velocity and concentration, respectively, for different source heights in the boundary layer. Note that there are more than 200 measurement points and the scatter in the values of $\tau_{u}$ is acceptable, with a clear trend in $\tau_{u}$ as a function of $z / \delta$. It is seen that $\tau_{u}$ increases up to $z / \delta \approx 0.2$, approximately remains constant between $0.2 \lesssim z / \delta \lesssim 0.5$ and then decreases up to the edge of boundary layer. Increase of $\tau_{u}$ adjacent to the wall is expected as the large-scale motions become prominent in the inner-region with increasing wall-normal height. In the region $z / \delta \gtrsim 0.5$, there is a decreasing trend of $\tau_{u}$ due to increasing external intermittency, i.e. length-scales associated with turbulent motions will be confined within regions of turbulent zones that are preceded and followed by non-turbulent regions. Since the relative population of turbulent zones decrease with increasing distance from the wall, $\tau_{u}$ decreases towards the edge of the boundary layer. Note that $\tau_{u}$ was also computed with data from single hot-wire (without any PID and the gas discharge tube), and the results are not different from what is presented in figure 5(a), although with less scatter.

Notice that the magnitude of $\tau_{u}$ is scaled with $\delta / U_{\infty}$, and the values are around unity, which suggests that $\tau_{u}$ is governed by the large-scale outer motions. Using a simplistic approximation of the integral times scales in shear flows to be of order of the inverse of the mean shear, or $1 / S \equiv$ $1 /(\mathrm{d} U / \mathrm{d} z)$, the usual logarithmic mean velocity profile $\left(U^{+}=(1 / \kappa) \ln \left(z^{+}\right)+A\right)$ approximation leads to a shear time-scale of $(1 / S) U_{\infty} / \delta=U_{\infty}^{+} \kappa(z / \delta)$ (where $\kappa=0.384$ is the von-Kármán constant), which is equal to 1.07 for $z / \delta=0.1$. Note that this time-scale turns out to be 0.97 if we approximate the velocity profile with a power-law $\left(U=U_{\infty}(z / \delta)^{\alpha}\right)$ with $\alpha=1 / 7$. These estimates are in the range of what we observe in figure $5(a)$, and as such valid in the region, $0.1 \lesssim z / \delta \lesssim 0.6$.

The variation of the integral time-scale of concentration $\tau_{c}$ is shown in figure $5(b)$, and is quite different from $\tau_{u}$. The collective trend of all data in figure 5(b) indicates that $\tau_{c}$ is high close to the 


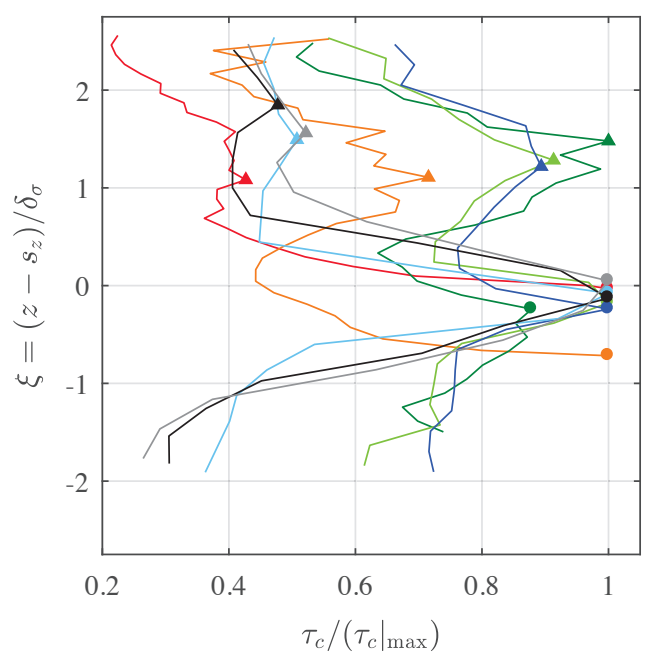

FIGURE 6. Normalised integral time-scale of $c\left(\tau_{c} /\left.\tau_{c}\right|_{\max }\right)$ for all source heights as a function of $\xi=\left(z-s_{z}\right) / \delta_{\sigma}$. The peak in $\tau_{c}$ closer to $\xi=0$ is shown by solid circles while the other peak is represented by solid triangles. Line colours as in figure 5 .

wall and near the edge of the boundary layer, whereas it does not vary significantly in the region $0.1<z / \delta<0.5$. These observations are consistent with those of Fackrell \& Robins (1982) for one ground level and one elevated source. It is clear that $\tau_{c}$ is influenced by background turbulence and the mean shear. Particularly, it should be noted that $\tau_{c}$ measured closer to the wall is significantly higher when the source is also in the vicinity of the wall. This behaviour could be due to small-scale turbulent motions close to the wall being effective in mixing and attenuating small-scale concentration fluctuations such that large-scale concentration patches remain, and they are associated with plume meandering. Comparing figures $5(a)$ and 5(b) shows that $\tau_{u}$ and $\tau_{c}$ have opposing overall trends, i.e. higher $\tau_{u}$ corresponds to relatively lower $\tau_{c}$ and vice-versa. This behaviour is again consistent with small velocity scales leading to better mixing and longer correlated scalar fields.

To put a perspective on the magnitude of $\tau_{c}$ in figure $5(b)$, it is worth considering the timescales associated with smallest turbulent motions, i.e. the Kolmogorov time-scale $\tau_{\nu}=\sqrt{\nu / \varepsilon}$, where $\varepsilon$ is the dissipation rate of kinetic energy and $\tau_{\nu}$ is sometimes considered to be the time of mixing or homogenisation (Tennekes \& Lumley 1972). Estimating $\varepsilon \approx U_{\tau}^{2} \mathrm{~d} U / \mathrm{d} z$, where $\mathrm{d} U / \mathrm{d} z$ is evaluated by assuming logarithmic profile for mean velocity $\left(U^{+}=(1 / \kappa) \ln z^{+}+\right.$A) leads to, $\tau_{\nu} U_{\infty} / \delta \approx U_{\infty}^{+} \sqrt{\kappa z^{+}} / \delta^{+}$. For $z / \delta=0.1$, we obtain $\tau_{\nu} U_{\infty} / \delta \approx 0.062$ (and 0.060 if we approximate $U$ with a power-law profile). As such, $\tau_{\nu} U_{\infty} / \delta$ is observed to be smaller than the typical $\tau_{c} U_{\infty} / \delta$ values reported in figure $5(b)$. This is perhaps not surprising considering that in a turbulent boundary layer, the dynamics are governed by the velocity fluctuations due to vortical motions, which are of the order of the Taylor microscale $\lambda$ (e.g. Chauhan et al. 2014a; de Silva et al. 2017). As such, if we consider the time-scale based on $\lambda$ and the fluctuating velocity $\left(\sigma_{u}\right)$, i.e. $\tau_{\lambda}=\lambda / \sigma_{u}$, and estimating $\lambda$ from $\varepsilon=15 \nu\left(\sigma_{u} / \lambda\right)^{2}$ leads to $\tau_{\lambda} U_{\infty} / \delta \approx U_{\infty}^{+} \sqrt{15 \kappa z^{+}} / \delta^{+}$. Then, for $z / \delta=0.1$, we obtain $\tau_{\lambda} U_{\infty} / \delta \approx 0.24$. This estimate of $\tau_{\lambda}$ is closer to the values of $\tau_{c}$ reported in figure $5(b)$, and shows that the time-scale of mixing is likely governed by velocity fluctuations.

Further, from figure 5, it is seen that the integral time-scale of velocity fluctuations is an order of magnitude higher than the integral time-scale of concentration, i.e. $\tau_{u} / \tau_{c}=O(10)$. This observation and its implication are later discussed in $\S 3.3$ along with discussion on spectra.

Closer examination of figure $5(b)$ indicates that two prominent peaks are present in $\tau_{c}$ profiles; the lower peak is closer to the plume centreline while the upper peak is approximately at a distance of $\delta_{\sigma}$ above the plume centreline. This feature is better illustrated in figure 6 , where normalised integral 
(a)

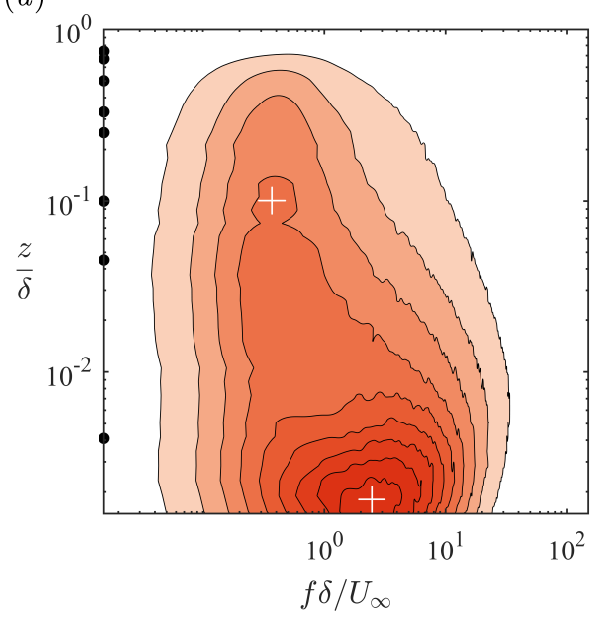

(b)

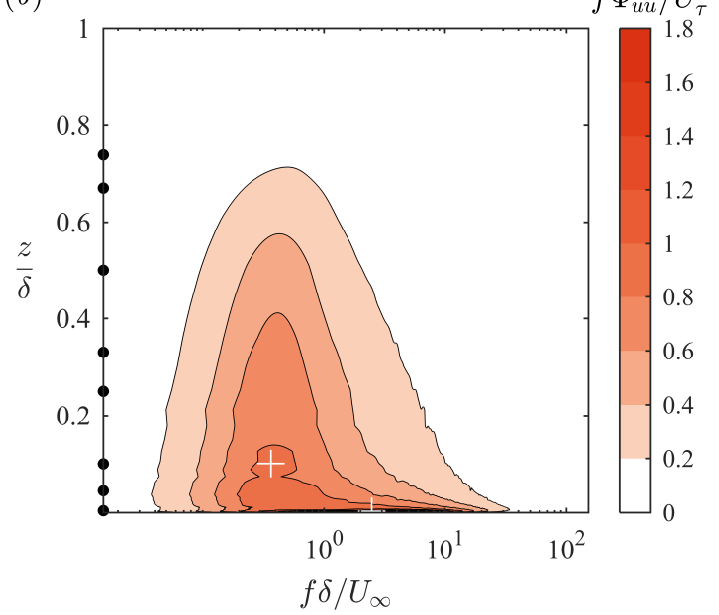

FIGURE 7. Contour map of pre-multiplied energy spectra of velocity fluctuations, $(a) \log -\log$ and $(b) \log$-linear scaling for abscissa and ordinate, respectively. Locations of inner and outer peaks in the energy spectra are indicated by ' + '.

time-scale $\tau_{c} /\left(\tau_{c, \max }\right)$ values are plotted against $\xi=\left(z-s_{z}\right) / \delta_{\sigma}$. In this coordinate system, $\xi=1$ corresponds to half-width (based on $\sigma_{c}$ ) above the centreline of a plume. The peak in $\tau_{c}$ near the centreline is most prominent for source heights, $s_{z} / \delta=32 / \delta^{+}, 350 / \delta^{+}, 0.5,0.67$, and $\delta_{99} / \delta$, whereas the second peak (near $\xi=1$ ) becomes relatively less prominent as $s_{z}$ increases. However, for sources located in the fully-turbulent regions, $s_{z} / \delta=0.1,0.25$ and 0.33 , both peaks are equivalent in magnitude. For sources located closer to the wall, i.e., $s_{z} / \delta \leq 0.1$, it is interesting to note that the peak in $\tau_{c}$ occurs below the centreline. This is likely because meandering of the plume below the centreline is restricted due to the presence of wall. In addition, the convection velocity is relatively small at these locations, which results in longer time for the scalar to be advected away, leading to higher magnitude of $\tau_{c}$ at locations below the plume centreline.

A similar result of double peak in $\tau_{c}$ was reported by Yee \& Biltoft (2004) in their full-scale atmospheric measurements of tracer gas dispersed through a large array of building-like obstacles. However, no explanation was provided for the existence of double peak. It should be noted that the present discussion is based on $\tau_{c}$ computed in Eulerian frame of reference. Unfortunately, it is extremely difficult to make direct measurements of Lagrangian time-scales. Typically, they are inferred from Eulerian measurements (see McComb 1990, for details) and it is usually hypothesised that Lagrangian and Eulerian correlation functions have similar shapes but different scales. However, we are unable to conclude whether or not a double peak exists in Lagrangian time-scales.

It is clear from figure 6 that $\tau_{c}$ has an asymmetric behaviour about the plume centreline. This asymmetry is a result of the variation in convection velocities as one moves across the boundary layer. Further the integral time-scales are higher away from the source height due to the (vertical) meandering or oscillating nature of the plume whereby it spends more time at the extreme ends. We shall elaborate on this phenomenon in $\S 5.1$ via a phenomenological model for velocity-concentration organisation.

\subsection{Pre-multiplied energy spectra}

We will consider the contribution of different frequencies to energy spectra of $u$ and $c$ in this section. The (one sided) spectral density $\Phi_{i i}$ and the auto-correlation function $R_{i i}$ are Fourier transform 


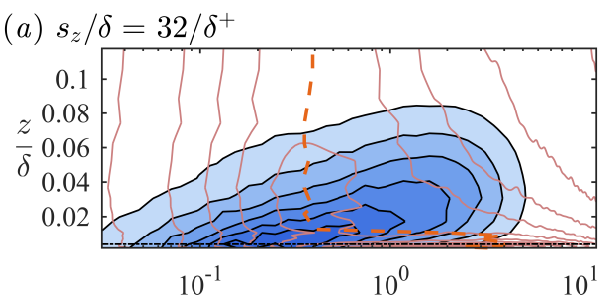

(c) $s_{z} / \delta=0.1$

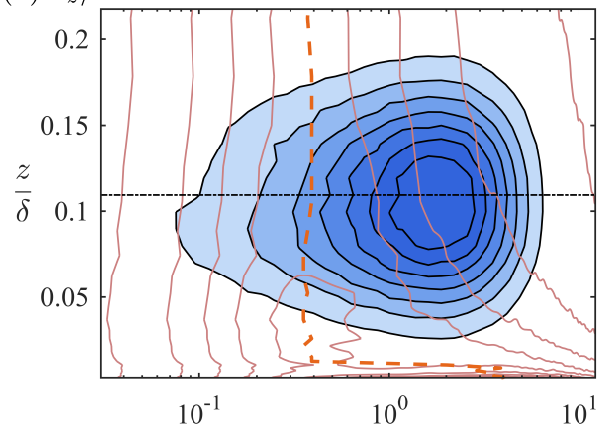

(e) $s_{z} / \delta=0.33$

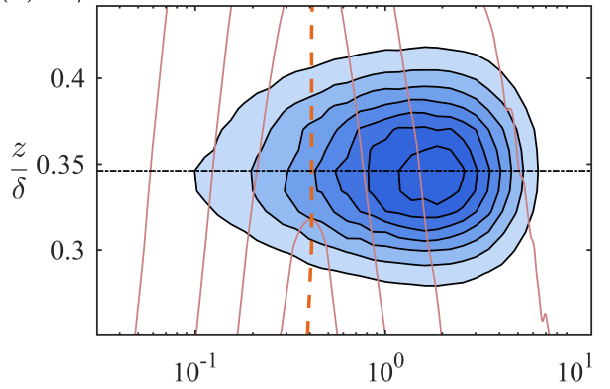

(g) $s_{z} / \delta=0.67$

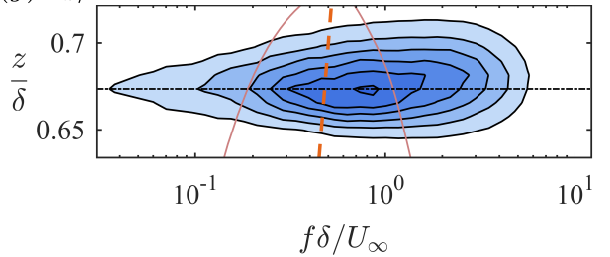

(b) $s_{z} / \delta=350 / \delta^{+}$

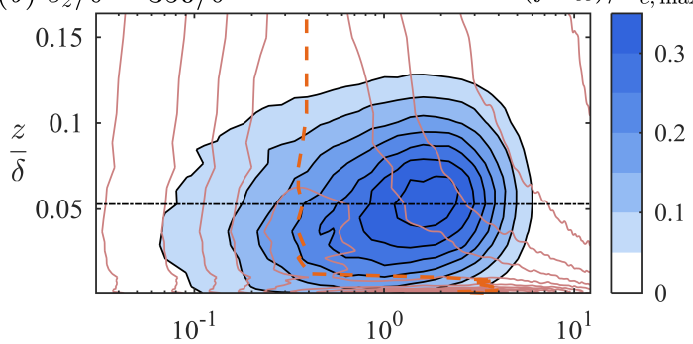

(d) $s_{z} / \delta=0.25$

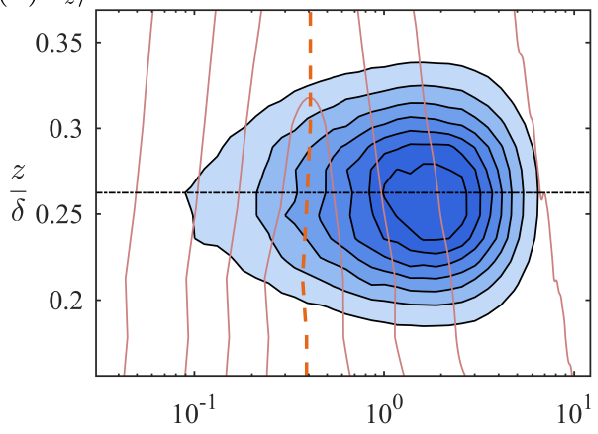

(f) $s_{z} / \delta=0.5$

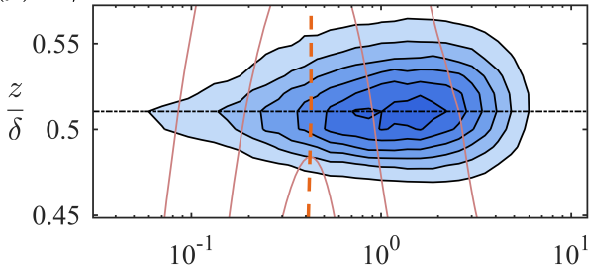

(h) $s_{z} / \delta=\delta_{99} / \delta$

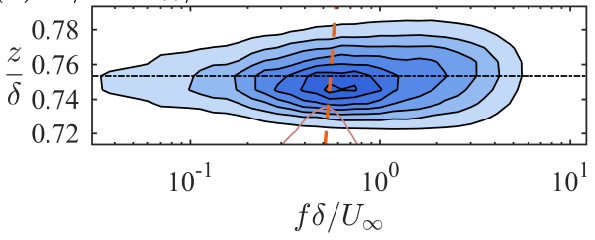

FIGURE 8. Contour maps of pre-multiplied spectra $\left(f \Phi_{c c} / \sigma_{c}^{2}\right.$, max $)$ of concentration fluctuations for different source heights. Here, $\sigma_{c}^{2}$, max represents the maxima in variance of concentration across the plume. Contours of $f \Phi_{u u} / U_{\tau}^{2}$ are shown in red. The red dashed lines represent the locus of local maxima of energy spectra of $u$ at different $z$-locations in the boundary layer. The horizontal black dot-dashed line in each subplot indicates the source location.

pairs, and hence $\Phi_{i i}$ is defined as,

$$
\frac{1}{2} \Phi_{i i}(f)=\frac{1}{2 \pi} \int_{-\infty}^{\infty} R_{i i}(t) e^{-j f t} \mathrm{~d} t, \quad i=u, c,
$$

where, $j$ represents the imaginary unit, and $t$ and $f$ are the corresponding time and frequency pairs. In order to facilitate visual integration of energy over certain frequency range, spectra is presented 
in the pre-multiplied form as $f \Phi_{i i}$ such that the variance, $\sigma_{i}{ }^{2}$ of a signal $i$ can be written as,

$$
\sigma_{i}{ }^{2}=\int_{0}^{\infty} \Phi_{i i}(f) \mathrm{d} f=\int_{0}^{\infty} f \Phi_{i i} \mathrm{~d} \ln f .
$$

We first examine pre-multiplied energy spectrogram of streamwise velocity $\left(f \Phi_{u u}\right)$ in figure 7 as functions of frequency $f$ and $z / \delta$. Note that figure $7(a)$ utilizes a log-log scale whereas $7(b)$ is with a linear-log scale. The well-known 'double-peak' in the spectrogram is also observed (see, e.g. Hutchins \& Marusic 2007a), and indicated by white '+' mark in the figure. The peak at higher frequency is associated with small-scale fluctuations and scales with viscous units $U_{\tau}^{2} / \nu$; whereas the one at lower frequency is a consequence of large-scale motions in the logarithmic region and above, and scales with $U_{\infty} / \delta$. As mentioned earlier, one cannot apply Taylor's hypothesis to concentration signal and thus we are restricted to examine $\Phi_{c c}$ as a function of frequency. This will enable us to make one-to-one comparison between $\Phi_{c c}$ and $\Phi_{u u}$.

Figure 8 shows the comparison of pre-multiplied energy spectrogram of concentration fluctuations $\left(f \Phi_{c c}\right)$ as filled contour maps for different source heights. In each plot, contours of $f \Phi_{u u} / U_{\tau}^{2}$ (same as in figure $7 b$ ) are overlaid as line plots. Also note that the ordinate scale of each panel is proportional although their vertical limits are different, providing a visual approximation of plume spread.

The most evident feature in figure 8 is that peaks in energy of $u$ (indicated by thick red dashed lines) at different heights are at a lower frequency compared to peak frequencies of concentration spectra. Peaks in spectra of concentration occur at frequency $f \sim 50-80 \mathrm{~Hz}$ compared to $f \sim$ $10-15 \mathrm{~Hz}$ for velocity spectra. Except for the source at ground level, the frequency at which the most energetic fluctuations in $c$ occur decreases as the source location is higher in the boundary layer. This behaviour is consistent with previous observation that the integral time-scales of concentration fluctuations $\left(\tau_{c}\right)$ are an order of magnitude smaller than those of velocity fluctuations $\left(\tau_{u}\right)$. The ground level source $\left(s_{z} / \delta=32 / \delta^{+}\right)$, however is an exception, where the peak-frequencies for $c$ spectra increase with increasing $z$. This behaviour reflects the steep decrease in $\tau_{c}$ observed near the wall in figure $5(b)$.

The lower peak frequency in the velocity spectra corresponds to a streamwise wavelength (by assuming local mean velocity as the convection velocity), $\lambda_{x} \sim 6 \delta-10 \delta$ consistent with the sizes of large-scale structures reported in literature. Overall, figure 8 suggests that large-scale velocity structures play only an indirect role, if any, on the structure of concentration in a boundary layer, where the concentration variation itself is at smaller spatial scale. This is expected, since in $\S 3.2$ it is seen that typical time-scales for concentration mixing are similar to those of small-scale velocity features, and hence have higher frequencies. A clearer understanding of interaction between largescale velocity and concentration is obtained by examining scalar flux $(\overline{u c})$ in the next section.

\section{Organisation of velocity and concentration in the flow}

Here we shall utilise the simultaneously measured velocity and scalar concentration to discuss velocity-scalar fluxes for different source heights, and their cross-spectral and phase relations.

\subsection{Streamwise scalar flux}

The gradient of streamwise scalar flux $\overline{u c}$ appears in the streamwise component of equation (1.1a) and is pertinent to downstream evolution of the plume. In practice, $\overline{u c}$ is neglected for being small in comparison to the mean transport $(\overline{U C})$ in equation $(1.1 a)$. To verify this, the streamwise turbulent flux $\overline{u c}$ is compared against $\overline{U C}$ across the plume released from a point source at $s_{z} / \delta=0.1$. It is found that $\overline{u c}$ is less than $4 \%$ of $\overline{U C}$, justifying the common approach of neglecting $\overline{u c}$ in equation $(1.1 a)$ for predicting $C$. However, the gradients of transverse $(\overline{v c})$ and vertical $(\overline{w c})$ fluxes in the respective directions are significant as they play a key role in spreading the plume. Further 

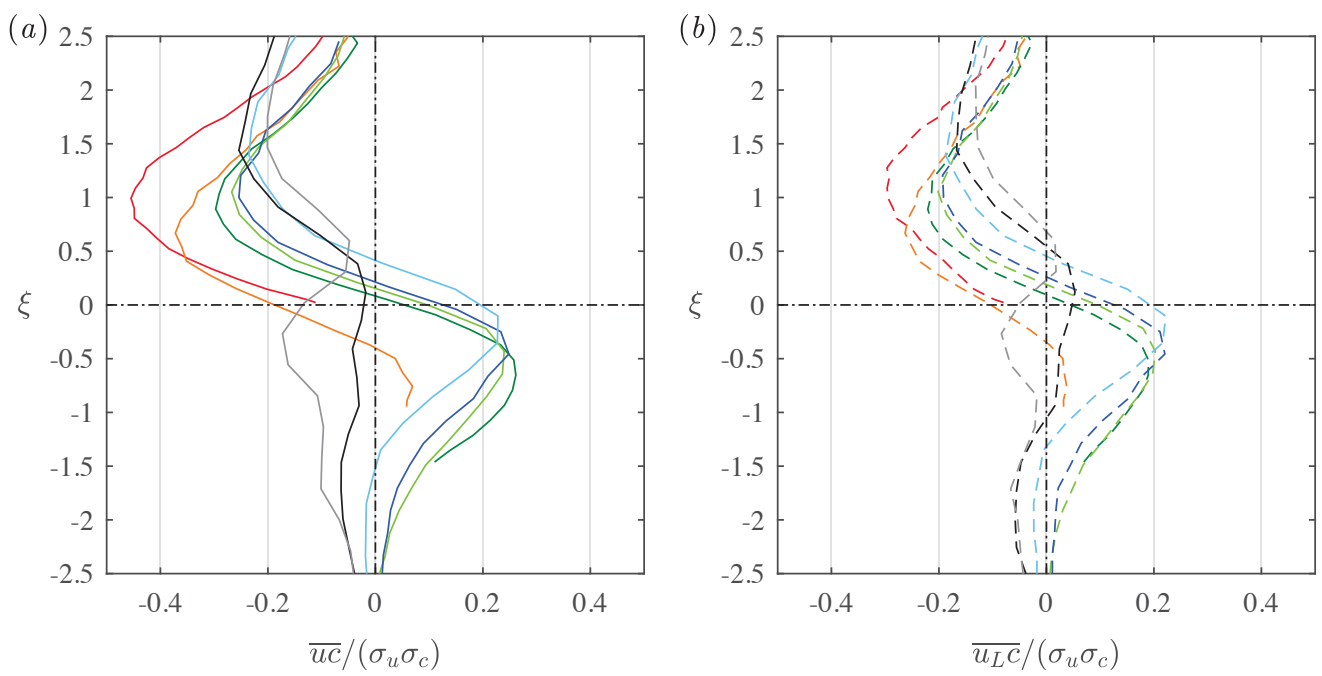

FIGURE 9. Comparison of (a) streamwise concentration flux normalised by the r.m.s of $u$ and $c$ for different source heights, and $(b)$ the contribution of large-scales of velocity to scalar flux. Line colours as in figure 5.

experiments aimed at characterising the gradients of these fluxes in the respective directions will be of great value.

The distribution of $\overline{u c}$ as a function of wall-normal distance is shown in figure $9(a)$ for all source heights. Results of $\overline{u c}$ are presented using the local ordinate $\xi=\left(z-s_{z}\right) / \delta_{\sigma}$ in order to examine characteristic behaviour of $\overline{u c}$ in the vicinity of plume centreline. For all source heights, $\overline{u c}$ is negative above the plume centreline $(\xi=0)$, while for $s_{z} / \delta \leq 0.5, \overline{u c}$ tends to be positive below $\xi=0$. Differing in trend from others, in the intermittent region, i.e. $s_{z} / \delta=0.67$ and $\delta_{99} / \delta, \overline{u c}$ is negative below the plume centreline.

In a laminar flow, the plume will spread only by the action of diffusion, whereas in a turbulent flow, a passive scalar will carried in the lateral and vertical directions by $v$ and $w$ fluctuations, respectively. In the wall-normal direction, positive $w$ fluctuations carry the scalar above the centreline and negative $w$-fluctuations below the centre line. Since the Reynolds stress, $\overline{u w}$ is negative throughout the boundary layer, negative $u$-fluctuations are correlated with positive $w$-fluctuations that are associated with upwards scalar transport, resulting in negative $\overline{u c}$ above the centreline. Similarly, positive $u$-fluctuations are correlated with negative $w$-fluctuations that are responsible for downwards scalar transport, leading to positive $\overline{u c}$ below the plume centreline. Thus, low-speed motions are associated with negative scalar flux above the plume centreline, and high-speed motions are associated with positive scalar flux below the centreline. This behaviour is supported by joint-probability density functions discussed in $\S 5.1$ and forms the basis of organisation of concentration patches in the flow relative to high- and low-speed velocity features.

Negative $\overline{u c}$ for $s_{z} / \delta=0.67$ and $\delta_{99} / \delta$, can be explained by considering influence of external intermittency in calculation of statistics. At $s_{z} / \delta=0.67$ the $\tilde{U}$-signal has about $50 \%$ of non-turbulent region and even higher fraction for the source at $\delta_{99} / \delta$. In the non-turbulent region, velocity is close to $U_{\infty}$ (typically about $0.95 U_{\infty}$, see Townsend 1976, and also Chauhan et al. 2014b). Therefore, the fluctuating streamwise velocity $u=\tilde{U}-U$ is typically negative in the turbulent region due to biased mean, resulting in a negative $\overline{u c}$. It is also found from a preliminary calculation that at the centreline for $s_{z} / \delta=0.67$, the turbulent zones in the signal contribute approximately $85 \%$ of $\overline{u c}$.

In section 3.3, the energy spectra of $u$ and $c$ revealed that the dominant frequency for velocity was smaller (indicating large scales) than that for the scalar. In order to understand the contribution of low-frequency velocity structures to the streamwise scalar flux $(\overline{u c})$, the instantaneous velocity is decomposed into large- and small-scales using a cut-off filter corresponding to wavelength of $1 \delta$ 


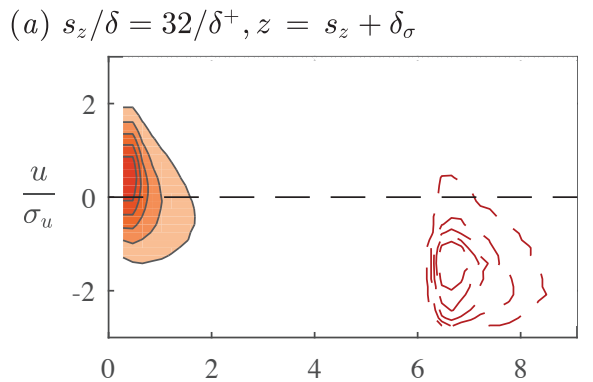

(b) $s_{z} / \delta=32 / \delta^{+}, z=s_{z}+\delta_{\sigma}$

(c) $s_{z} / \delta=0.1, z=s_{z}+\delta_{\sigma}$

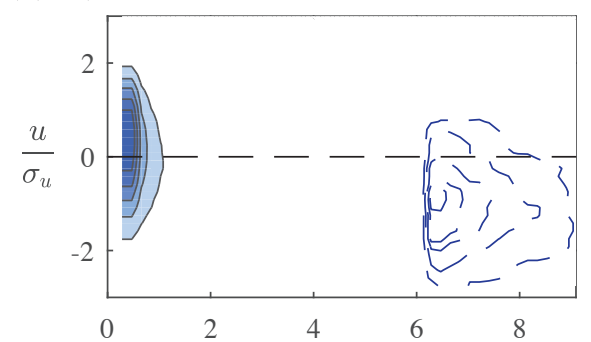

(e) $s_{z} / \delta=0.1, z=s_{z}-\delta_{\sigma}$
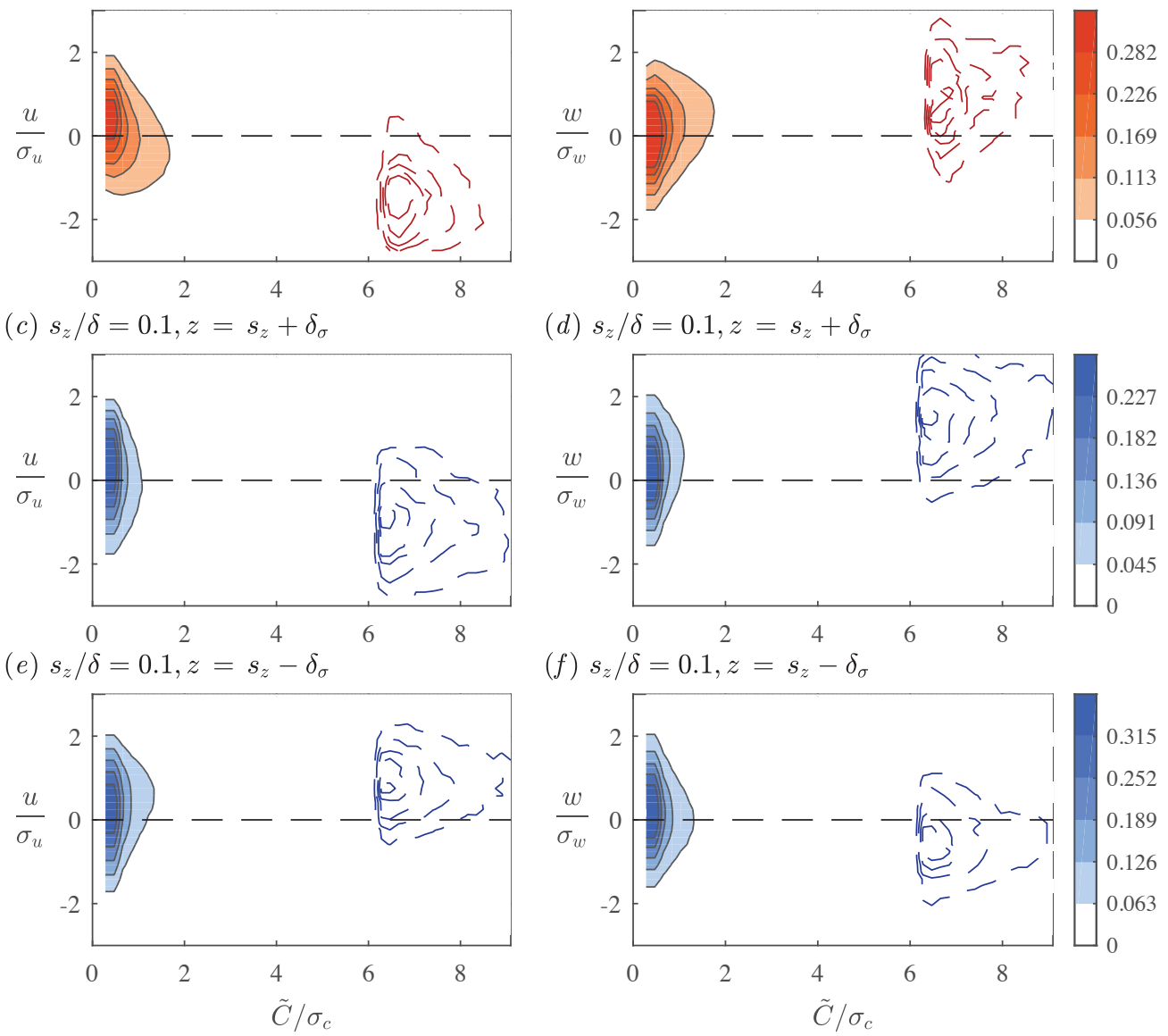

(d) $s_{z} / \delta=0.1, z=s_{z}+\delta_{\sigma}$

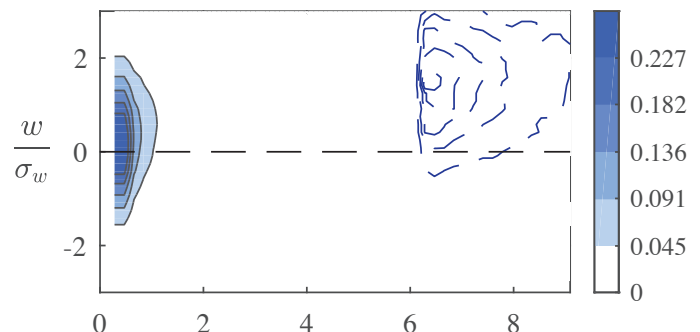

(f) $s_{z} / \delta=0.1, z=s_{z}-\delta_{\sigma}$

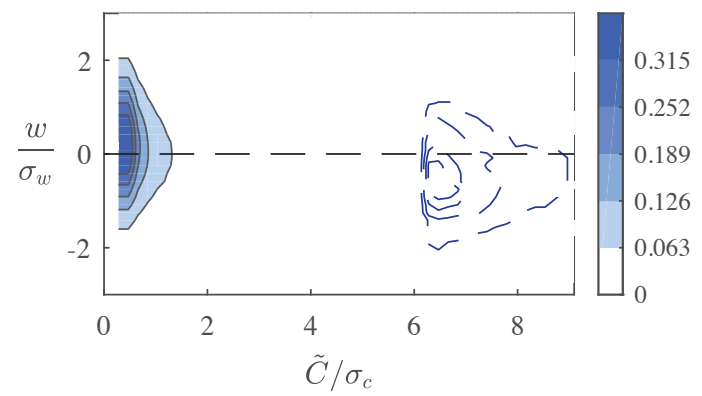

FIGURE 10. Joint probability density functions of $u$ and $c$, and $w$ and $c$ at a location, $(a, b) s_{z}+\delta_{\sigma}$ for $s_{z} / \delta=32 / \delta^{+} ;(c, d) s_{z}+\delta_{\sigma}$ and $(e, f)$ at $s_{z}-\delta_{\sigma}$ for $s_{z} / \delta=0.1$. Filled contours show the j-pdf of $u$ and $\tilde{C}$ without any threshold on concentration, while the dashed lines represent j-pdf of $u$ and $\tilde{C}$ at high concentration. Here each adjacent plot share the same color-bar. Contour lines have the same levels as in the color-bar of filled contours.

such that $u=u_{L}+u_{S}$, where $u_{L}$ and $u_{S}$ are the large- and small-scale velocity components, respectively. The choice of $1 \delta$ as the filter cut-off is adopted from previous studies of Hutchins \& Marusic (2007b); Mathis et al. (2009) in smooth-wall zero-pressure-gradient turbulent boundary layer. The resulting large-scale concentration flux $\overline{u_{L} c}$ is plotted in figure $9(b)$, noting that $\overline{u c}=\overline{u_{L} c}+\overline{u_{S} c}$. It is clear that $\overline{u_{L} c}$ contributes significantly to the magnitude of $\overline{u c}$, a conclusion that could not be drawn from spectra alone. The strong $\overline{u_{L} c}$ correlation implies that although high concentration regions in the flow occur at small smaller spatial scales, they are effectively transported by large-scale motions associated with coherent structures. Such large-scales coherent structures that are wellidentified in zero-pressure gradient turbulent boundary layers are long meandering super-structures (see Hutchins \& Marusic 2007a), counter-rotating roll-modes (e.g. Hutchins \& Marusic 2007b) and vortex packets (e.g. Christensen \& Adrian 2001). So far, our observations suggest that both lateral and vertical shear stress events ( $\overline{u v}$ and $\overline{u w}$, respectively) associated with large-scale motions are responsible for the instantaneous spread of plume and its meandering behaviour. 


\subsection{Joint-probability density functions}

In this section, we will study the role of $w$-fluctuations in the vertical transport of scalar. For this, we conducted additional experiments using a Cobra probe and PID to simultaneously measure all three velocity components as well as concentration. Although, measurements are available from eight source heights, only results for one ground level source and one elevated source in the log-region are presented here. It has been observed that the results at other source heights are similar to those presented here.

Joint-probability density functions (j-pdf, hereafter) of instantaneous concentration $\tilde{C}$ and either of three velocity fluctuations $(u, v, w)$ are computed. For the ground level source, $s_{z} / \delta=32 / \delta^{+}$, figures 10(a) and 10(b) plot the contours of j-pdf of $u$ and $c$, and $w$ and $c$, respectively. These $\mathrm{j}$-pdf are obtained at a location $z=s_{z}+\delta_{\sigma}$, i.e. one half-plume-width above the centreline. The j-pdf of $v$ and $c$ is found to be symmetric about $v=0$ and hence is not shown here. In contrast to conventional $\mathrm{j}$-pdf, we have used instantaneous concentration $(\tilde{C})$ rather than fluctuating concentration $(c)$ to always have a positive value for concentration. By adopting this approach, it is easier to quantify the contribution of positive and negative velocity fluctuations $(u, v$ and $w)$ to high instantaneous concentration events. Further, the j-pdfs between velocity and concentration fluctuation $(c)$ are qualitatively similar to the results presented in figure 10 and can be obtained simply by shifting the horizontal axis to the left by a value equal to the mean concentration.

In each plot of figure 10, filled contours show the j-pdf obtained from full signal, whereas the dashed contour lines show the corresponding j-pdfs calculated from portions of the signals when the instantaneous concentration is above a certain threshold (here it is taken as $6 \sigma_{c}$ ). Further, it is important to note that the levels of filled contours and the dashed contours are represented by the same colour bar. In this conditional representation, dashed contours represent combined probability of velocity and concentration fluctuations when concentration is high. It is observed that $\mathrm{j}$-pdf of $u$ and $\tilde{C}$ computed from Cobra probe and PID measurements is in excellent agreement with those obtained from single hotwire and PID experiments. Further it is seen that for the ground source and measurements above the plume centreline, $u$-fluctuations are negative when $\tilde{C}$ is high (figure $10 a$ ), and this corresponds well with positive $w$-fluctuations and high $\tilde{C}$ (cf. figure $10 b$ ). Thus, highconcentration events above the plume source height are associated with negative $u$ and positive $w$, with an overall negative $\overline{u w}$.

These observations are also true in the case of elevated sources as evident from figure $10(c, d, e$ and $f$ ) for source at $s_{z} / \delta=0.1$. Here, the j-pdfs are obtained from two locations that are at a distance of half plume-width on either side of the plume centreline, i.e. $z=s_{z} \pm \delta_{\sigma}$. Above the plume centreline the sign-preference for $u$ - and $w$-fluctuations remain the same for the elevated source as it was for the ground level source. However, below the plume centreline, high-concentration events are concurrent with positive $u$-fluctuations and negative $w$-fluctuations. The overall contribution of these events is still negative towards mean $\overline{u w}$. These observations support our hypothesis of local transport by wall-normal motions, i.e. $w$-fluctuations and associated shear stress $(\overline{u w})$ are responsible for vertical spread of the plume.

\subsection{Velocity-concentration cross-correlation}

The cross-correlation function $R_{u c}$ between the fluctuating $u$ and $c$, is defined as,

$$
R_{u c}(\mathbf{x}, \Delta t)=\overline{u(\mathbf{x}, \mathbf{t}) c(\mathbf{x}, t+\Delta t)} .
$$

Similar to $R_{i i}$ in $\S 3.2, R_{u c}$ is different for different source heights and in our single-point measurements varies with $z$. Results of $R_{u c} /\left(\sigma_{u} \sigma_{c}\right)$ are presented as contour maps in figures 11 for the eight source heights. Note that time shift $\Delta t$ is normalised by $\delta / U_{\infty}$ and $\Delta t=0$ corresponds to distributions of $\overline{u c}$ in figure 9. Continuing from observations in figure 9, contours for positive correlation are below the centreline while contours for negative correlation are above the centreline. The contours also appear to be elongated along the horizontal as a foot-print of large-scale velocity 
(a) $s_{z} / \delta=32 / \delta^{+}$

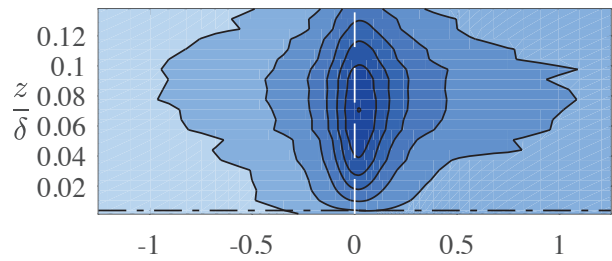

(c) $s_{z} / \delta=0.1$

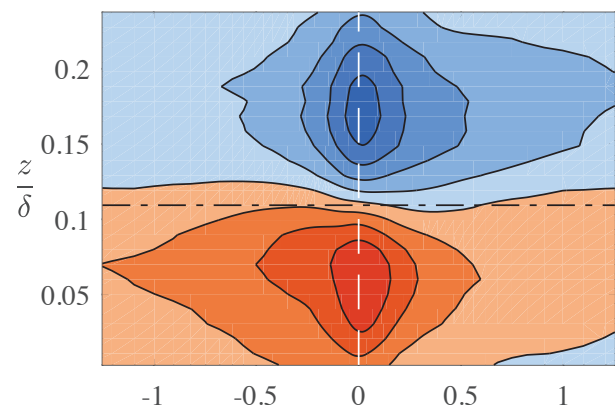

(e) $s_{z} / \delta=0.33$

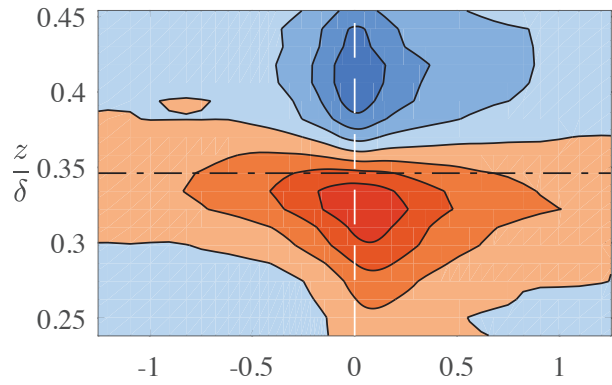

(g) $s_{z} / \delta=0.67$

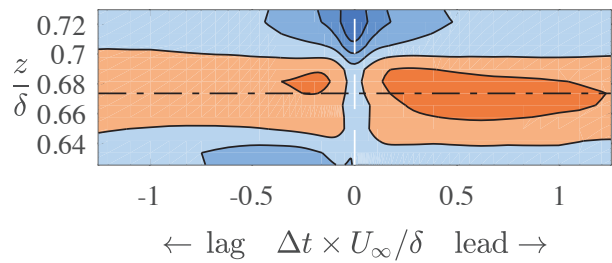

(b) $s_{z} / \delta=350 / \delta^{+}$

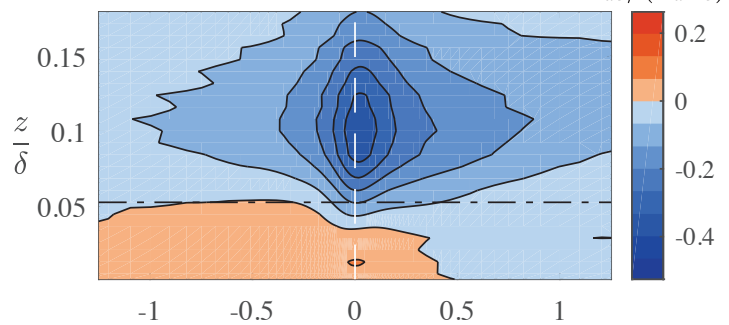

(d) $s_{z} / \delta=0.25$

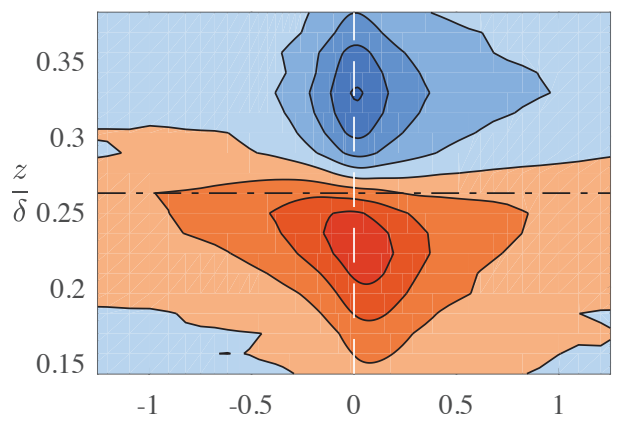

(f) $s_{z} / \delta=0.5$

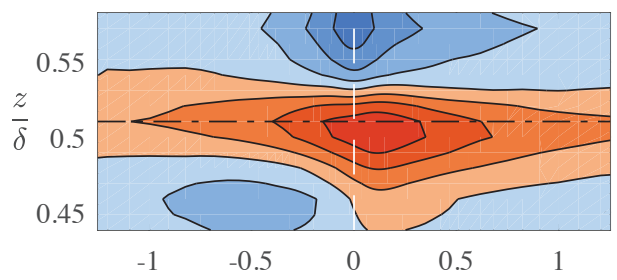

(h) $s_{z} / \delta=\delta_{99} / \delta$

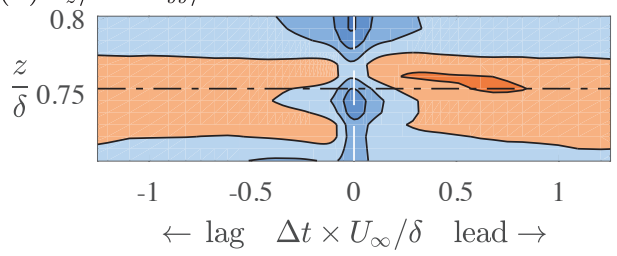

FIGURE 11. Contour maps of cross-correlation coefficient, $R_{u c} /\left(\sigma_{u} \sigma_{c}\right)$. The horizontal black dot-dashed line in each subplot indicates the source location.

structures that are associated with high $\overline{u c}$. It is clear that the correlation between $u$ and $c$ declines rapidly within a time-scale that corresponds to a physical distance of approximately $1 \delta-2 \delta$ if one considers freestream velocity $U_{\infty}$ as the convection velocity. Thus, the elongated contours in figure 11, suggest that although concentration events are relatively smaller than large-scale turbulent motions, they correlate well over extents of $O(1 \delta)$. Therefore, large-scale coherent features are of significance for understanding the mechanisms of scalar-transport.

Further, for source heights up to and including $s_{z} / \delta=0.5$, there is a noticeable asymmetry in negative $R_{u c}$ above the plume centreline, i.e. the contours extend towards positive $\Delta t$. In contrast, (for $s_{z} / \delta=350 / \delta^{+}, 0.1$, and 0.25 ), the asymmetry for the positive contours is on the opposite side, i.e. the contours extend towards negative $\Delta t$. In general, it is inferred that above the source 
(a)

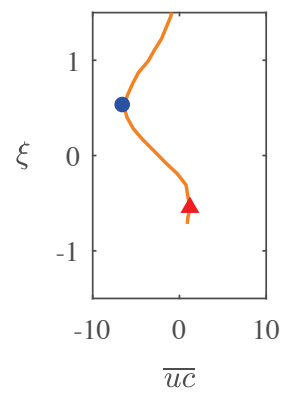

$(d)$

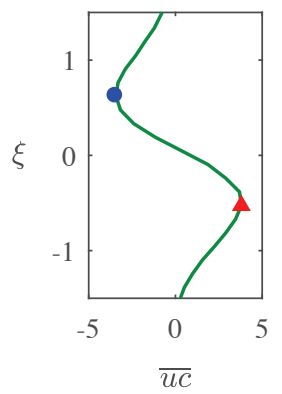

(b)

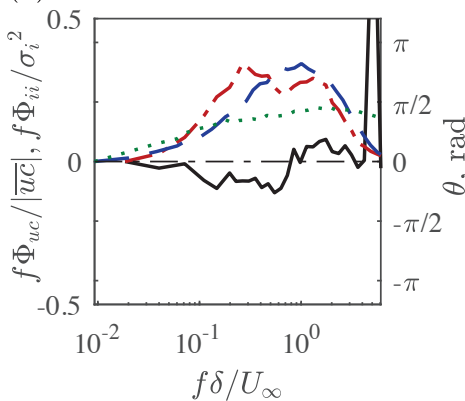

(e)

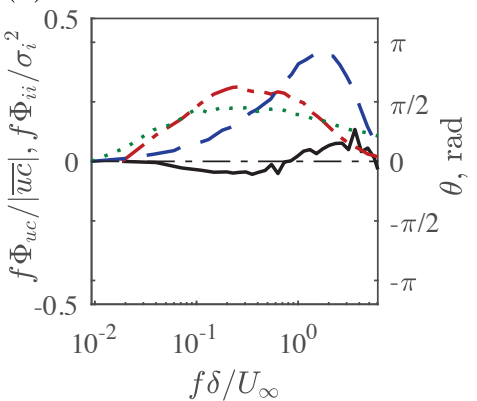

(c)

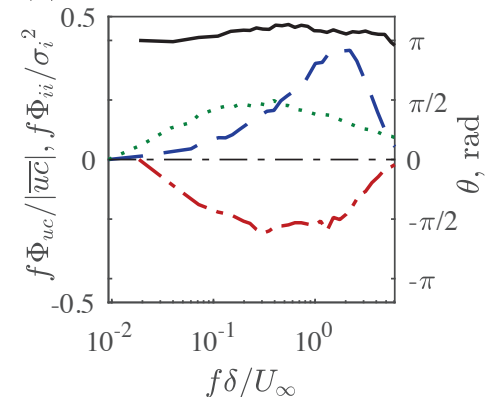

(f)

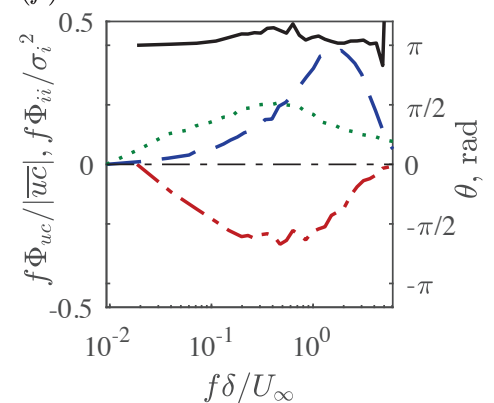

FiguRE 12. $(a, d)$ - Distribution of $\overline{u c}$ as a function of $\xi$ for two source heights, $s_{z} / \delta=350 / \delta^{+}$and $s_{z} / \delta=0.1$, respectively. In other plots: $f \Phi_{u u} \sqrt{u^{2}}$ - (green) dotted line; $f \Phi_{c c} / \overline{c^{2}}$ - (blue) dashed line; $f \Phi_{u c} /|\overline{u c}|$ - (red) dot-dashed line and $\theta$ - (black) solid line. $(b, e) \overline{u c}$ is maximum below $\xi=0$ and $(c, f)$ $\overline{u c}$ is minimum above $\xi=0$.

height, large-scale low-speed velocity structure has a lead over regions of significant concentration. Similarly, below the source height, large-scale high-speed velocity regions are lagging behind peak concentration events.

\subsection{Pre-multiplied cross spectral density}

In this section, further evidence is sought for the relative lead/lag observed between velocity and concentration in figure 11 by examining cross-spectral density. The cross-correlation function, $R_{u c}$ and the cross-spectra $\Gamma_{u c}$ are Fourier transform pairs related as,

$$
\frac{1}{2} \Gamma_{u c}(f)=\frac{1}{2 \pi} \int_{-\infty}^{\infty} R_{u c}(t) e^{-j f t} \mathrm{~d} t .
$$

The quantity $\Gamma_{u c}(f)$ is in general complex, and it can be expressed as $\Gamma_{u c}(f)=\Phi_{u c}(f)+j \Psi_{u c}(f)$ where, $\Phi_{u c}$ and $\Psi_{u c}$ are the magnitudes of real and imaginary components. Based on this definition of $\Gamma_{u c}$, we can obtain the phase angle $(\theta)$ between $u$ and $c$ from the ratio of imaginary and real parts as,

$$
\theta(f)=\tan ^{-1}\left\{\frac{\Psi_{u c}(f)}{\Phi_{u c}(f)}\right\} .
$$

Given the definition of $R_{u c}$ in equation (4.1), a positive $\theta$ implies that $u$ leads $c$, and a negative $\theta$ will indicate $u$ lags $c$. Further, it is only the real component of $\Gamma_{u c}(f)$, i.e. $\Phi_{u c}$, that contributes to scalar flux $\overline{u c}$ (a real quantity), and $\overline{u c}$ can be shown to be equal to the area under the pre-multiplied form of $\Phi_{u c}$ as $\overline{u c}=\int_{0}^{\infty} f \Phi_{u c} \mathrm{~d} \ln f$. Henceforth, the discussion will be based on the real component of cross-spectra in the pre-multiplied form $\left(f \Phi_{u c}\right)$.

For brevity, we will discuss the cross-spectra at two distinct points in profiles for $\overline{u c}$ when the source is located at $s_{z} / \delta=350 / \delta^{+}$and $s_{z} / \delta=0.1$. These two distinct points correspond to 
the locations where $\overline{u c}$ is either maximum on minimum on either side of the plume centreline and indicated by solid symbols in figures $12(a)$ and $12(d)$. The other four panels in figure 12, i.e. $(b)$, $(c),(e)$ and $(f)$ plots $f \Phi_{u u} \sqrt{u^{2}}$ (dotted line), $f \Phi_{c c} / \overline{c^{2}}$ (dashed line), $f \Phi_{u c} /|\overline{u c}|$ (dot-dashed line) and the corresponding phase angle $(\theta$, solid line) between $u$ and $c$ plotted together as a function of frequency. Figure 12(b) corresponds to positive maxima (solid triangle) and figure 12(c) corresponds to negative minima (solid circle) of $\overline{u c}$ in figure 12(a). Similarly figure 12(e) corresponds to positive maxima (solid triangle) and figure $12(f)$ corresponds to negative minima (solid circle) of $\overline{u c}$ in figure $12(d)$

Figure 12(e) shows that the peak in $\Phi_{u u}$ occurs at lower frequency than $\Phi_{c c}$ as expected from discussion in $\$ 3.3$. The cross-spectra, which receives contributions from both $u$ and $c$, however, peaks at a frequency which is closer to the peak frequency of $u$. Consistent with the results of figure $9(b)$ where we observed that large-scale velocity fluctuations, $u_{L}$ contributes significantly to $\overline{u c}$, $\Phi_{u c}$ indeed mimics $\Phi_{u}$ closer than $\Phi_{c}$, and obtains most of its contributions from lower frequencies of $u$. This suggests that indeed the scalar is 'passive' for its contributions to the flux. This feature, however, is not observed for the measurement point close to the wall (figure $12 b$ ), where dominant frequencies for $\Phi_{u c}$ are closer to those for $\Phi_{c}$ and there the concentration length-scales dominates. This is due to the wall effect, where there is zero flux of concentration.

Further, in figure $12(c \& f)$, corresponding to $\overline{u c}<0$, it is evident that the magnitude of $f \Phi_{u c} /|\overline{u c}|$ is negative across the entire range of frequency and more importantly, the phase angle is close to $\pi$. This implies that $u$ and $c$ are out of phase, which contributes to negative scalar flux in that region. Also, the phase $\theta$ is positive, which tells us that $u$ leads $c$. Likewise, in the positive flux region below the plume centreline (figures $12 b \& e$ ), it is seen that $\theta$ is predominantly close to zero suggesting that $u$ and $c$ are mostly in phase contributing to the positive value of $\overline{u c}$. Also, for most of the frequencies which contributed to $\overline{u c}$, the phase is negative (slightly below 0 ) showing that $u$ is lagging $c$. These observations substantiate the proposition that below the centreline of plume, high speed regions are lagging high concentration regions and contribute to the positive concentration flux, while above the centre line low momentum structures lead high concentration regions and contribute to negative flux. The phase information obtained via cross-spectra analysis lends support to the lead/lag between $u$ and $c$ observed in the two-dimensional cross-correlation maps presented in figure 11. Furthermore, figures 11 and 12 represent the same information; however, the former in physical space while the latter in spectral space.

\section{Discussion and conclusions}

\subsection{A phenomenological model}

In previous sections statistical results for the integral time-scales, scalar-flux, cross-correlation and relative organisation of instantaneous velocity and concentration in the flow were discussed. It becomes necessary now to collate those results and develop a unified view of flow mechanisms that support our observations. This is achieved through a simplified physical model of the plume as presented figure 13, which shows a plume emitted from a source that disperses about its local centre (i.e. become wider in size) and meanders or oscillates roughly about the centreline. Note that this model is only valid for sources located in the fully turbulent region (i.e. say below $z / \delta \approx 0.6$ ) and not for the ground level source. The wall reduces meandering effect and due to the zero flux condition at the wall, results in higher local concentration levels (e.g. Csanady 1973). We also would like to mention that flow visualisations (in the same set-up using visible smoke instead of the tracer gas) clearly shows that plume meanders, although in the schematic the extent of meander is accentuated. The meandering plume model has been used in the past to provide quantitative information on statistics of scalar (e.g. Gifford 1959), however, not for studying the velocity-scalar interaction, which is our aim here. Here three main results are explained below using this model:

(a) In figure $9(a)$, it is found that scalar-flux $\overline{u c}$ is negative above the plume centreline and positive 
below. As indicated in figure 13, the meandering, which is caused by the $w$-fluctuations, carries the plume up $(w>0)$ or down $(w<0)$. The joint-probability distributions in figure 10 attest to this behaviour for high concentration levels. Since the Reynolds shear stress $\overline{u w}<0$, when $w$ is negative, $u$ is likely to be positive and vice-versa. Due to meandering, given the assertion that $u$ is negative and positive when the plume is above and below, respectively, the flux $\overline{u c}$ has to follow the sign of $u$-fluctuation (since $\tilde{C}$ is positively skewed), hence the resultant distribution in figure $9(a)$.

(b) A 'double-peak' in the wall-normal distribution of $\tau_{c}$ was observed in figure 6. Also, there is a lack of secondary peak below the plume centreline. Given similar mixing characteristics, if the plume spends more time at a location, the tendency is to have a larger $\tau_{c}$. Therefore, it is not surprising that one peak in $\tau_{c}$ appears close to the centreline, because although the plume is meandering, it is still primarily at the centreline. However, the meandering implies that the plume will also spend more time at the edges (although an exaggeration, in manner similar to pendulum). Assuming that the local convection velocity of scalar is the same as the local flow velocity (since it is a passive scalar), the plume above the centreline is convected with motions that have $u<0$ and thus spends more time at the probe. Hence giving rise to the second peak in $\tau_{c}$. Furthermore, when the plume is below the centreline the corresponding $u$-fluctuations that convects it are positive, and therefore a relatively smaller time is spent at the probe, compared to when the plume is above the centreline hence the asymmetry in the $\tau_{c}$ distribution about the centreline.

(c) In figure 11, say for $s_{z} / \delta=0.1$ corresponding to figure 11(c), we observed lead for negative (blue) contours of $R_{u c}$ above the centreline whereas a lag for positive (red) contours below the centreline. This suggests that the low-speed structures lead the plume in the region above the centreline while high-speed structures are lagging behind the plume below its centreline. Let us consider, $w>0$ (ejection-like events) indicated in figure 13, that cause the plume to meander above the centreline. With such events, the gradient $\partial w / \partial z>0$ (for the plume to spread upwards) and from (2D) continuity equation, it can be inferred that $\partial u / \partial x<0$. The two conditions: $u<0$ and $\partial u / \partial x<0$ imply that the tail of large-scale low-speed structure is well correlated with concentration fluctuations above the centreline of the plume, i.e. peak negative $u$ fluctuation events lead peak concentration events. Likewise, sweep-like events with $w<0$ and $\partial w / \partial z>0$ convect the plume downwards. The associated two conditions for streamwise velocity fluctuations, $u>0$ and $\partial u / \partial x<0$ imply that the frontal portion of a high-speed structures are well-correlated with concentration fluctuations below the centreline of a meandering plume.

In order to provide physical evidence for the role of transverse and vertical velocity fluctuations in plume meandering, we have conducted smoke visualisation of the plume released at $z / \delta=0.2$. One of the time instances is shown in the inset of figure 13, which clearly shows the meandering behaviour of the plume. It is found that the wavelength of the meander is approximately $0.5 \delta$ at this instant. From the recent measurements in high Reynolds number turbulent boundary layers (Baidya et al. 2017), the dominant streamwise length-scale of $w$-fluctuations is found to scale as $\lambda_{x_{w}} \approx 2 z$, which for $z=0.2 \delta$ gives $\lambda_{x_{w}} \approx 0.4 \delta$. This indicates that the meandering length-scale is of the same order as that of vertical velocity length-scale, which is suggestive that the vertical velocity plays a key role in plume meandering, in support of our physical model.

An obvious question that follows is how does the time-scales of plume meandering compare against integral time-scale of concentration, $\tau_{c}$, previously discussed in $\S 3.2$. In order to estimate the time-scale of plume meandering, we need an appropriate velocity scale for its travel in the transverse or lateral direction, which is possible only by Lagrangian tracking of scalar, and we have not performed such a measurement. Anyhow, to compare with the Eulerian estimate $\tau_{c}$, the meandering time of the plume can be indirectly estimated by two approaches. First, we are interested in the time over which the meandering plume goes past a stationary probe, denoted here as $\tau_{m_{E}}$. Here we consider the local convection velocity $U(z)$ to be the appropriate velocity scale. For $z / \delta=0.2$, $U(z) \approx 8.56 \mathrm{~m} / \mathrm{s}$ and with the meandering streamwise length-scale to be $0.5 \delta, \tau_{m_{E}} U_{\infty} / \delta \approx 0.6$. This is about three times the integral concentration time-scales $\tau_{c}$ (c.f. $\tau_{c} U_{\infty} / \delta \approx 0.2$ in figure $5 b$ ). 


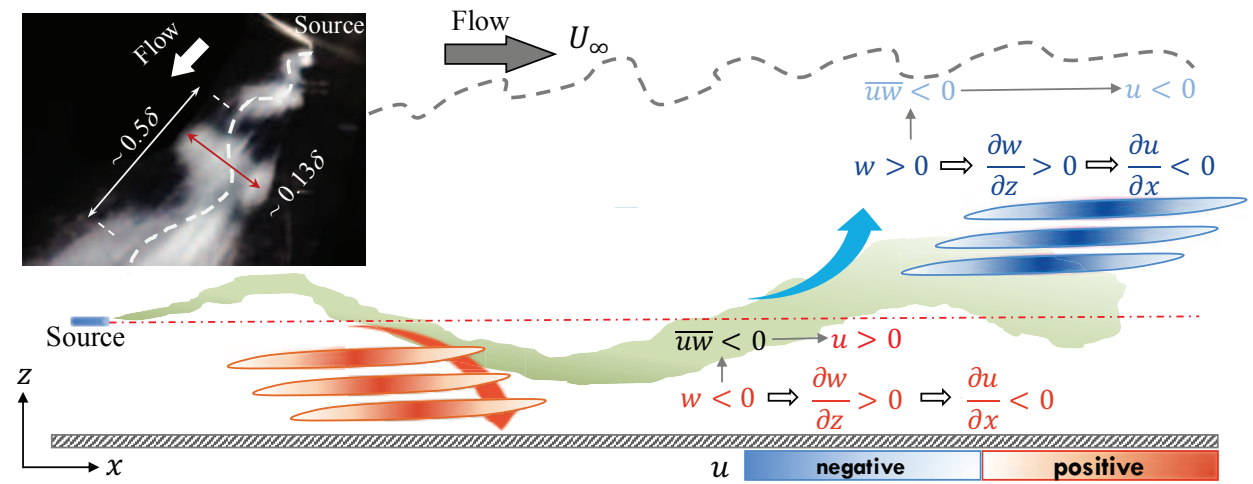

FIGURE 13. Relative arrangement of large-scale velocity structures around a decaying meandering plume shown in green. Low- and high-speed structures are depicted as blue and red regions in the schematic, respectively. Inset: A flow visualisation of the plume released at $s_{z} / \delta \approx 0.4$, and captured by a 'Go-Pro' series wide angle view camera. Note that the direction of flow is indicated by the white arrow and the meandering of plume is shown by a dashed line.

A second approach, and perhaps a more relevant meandering time-scale closer to the Lagrangian viewpoint is the time-scale at which the plume meanders up and down. For the corresponding velocity scale, it is justified to consider the root mean square of $w$-fluctuations $\left(\sigma_{w}\right)$ as a first approximation since the scalar is passive. Further, the appropriate length-scale is the amplitude of the meandering in the $z$-direction, here it is considered as $2 \delta_{\sigma}=0.13 \delta$ (c.f. figure 3 , which is further confirmed via smoke visualisation in figure 13). As such, the time-scale of plume meandering in Lagrangian reference frame can be approximated as $\tau_{m_{L}}=0.13 \delta / \sigma_{w}\left(\sigma_{w} U_{\tau} / \nu \approx 1.4\right.$ at $z / \delta=$ 0.2 (Baidya et al. 2017)), which in non-dimensional form becomes $\tau_{m_{L}} U_{\infty} / \delta \approx 2.5$. This estimate is an order of magnitude larger than $\tau_{c} U_{\infty} / \delta$. Therefore, it is reasonable to interpret that meandering occurs at a much longer time-scale in comparison to the integral time-scale $\tau_{c}$. Admittedly the proposed velocity-concentration model is simplified and perhaps preliminary, and further work is required to make it quantitative. Nevertheless, it provides a consolidated summary of observations made in a consistent manner.

\subsection{Conclusions}

High quality measurements (with good spatial and temporal resolution) of velocity and concentration fluctuations downstream of a plume released at different heights in a turbulent boundary layer are reported here with a specific aim of understanding the role of velocity structures in the local transport of scalar. A comparison of mean and r.m.s profiles for different source heights in the boundary layer suggest that all elevated plumes can be fitted to the reflected-Gaussian distribution while ground level plumes exhibit an exponential distribution. This is found to be consistent with the previous studies of Fackrell \& Robins (1982) and Nironi et al. (2015). Temporally resolved concentration data enabled us to compute integral time-scales of concentration across the plume and it is observed that the integral time-scales of concentration fluctuations are nominally $O(10)$ smaller than those of velocity fluctuations, implying that concentration structures have shorter length-scales. Further, the streamwise concentration flux is found to be negative and positive above and below the plume centreline, respectively. Cross-correlation results and the phase relationship between velocity and concentration fluctuation indicates that there is a predominant phase angle of $\pi$ between $u$ and $c$ above the plume centreline where $u$ leads $c$, while this trend gets reversed below the centreline. All these observations are explained using a physical model that is based on arrangement of instantaneous velocity and scalar structures. 


\section{REFERENCES}

Adrian, R. J., Meinhart, C. D. \& Tomkins, C. D. 2000 Vortex organization in the outer region of the turbulent boundary layer. J. Fluid Mech. 422, 1-54.

Antonia, R. A., DANH, H. Q. \& PRABhU, A. 1977 Response of a turbulent boundary layer to a step change in surface heat flux. J. Fluid Mech. 80 (1), 153-177.

ARYA, S. P. S. 1975 Buoyancy effects in a horizontal flat-plate boundary layer. J. Fluid Mech. 68 (2), 321-343.

Baidya, R., Philip, J., Hutchins, N., Monty, J. P. \& Marusic, I. 2017 Distance from the wall scaling of turbulent motions in wall-bounded flows. Phys. Fluids 29 (2), 020712.

Chatwin, P. C. \& Sullivan, P. J. 1979 The relative diffusion of a cloud of passive contaminant in incompressible turbulent flow. J. Fluid Mech. 91 (2), 337-355.

Chauhan, K., Philip, J. \& MARusic, I. 2014a Scaling of the turbulent/non-turbulent interface in boundary layers. J. Fluid Mech. 751, 298-328.

Chauhan, K. A., Nagib, H. M. \& Monkewitz, P. A. 2009 Criteria for assessing experiments in zero pressure gradient boundary layers. Fluid Dyn. Res. 41, 021404.

Chauhan, K. A., Philip, J., De Silva, C. M., Hutchins, N. \& Marusic, I. $2014 b$ The turbulent/nonturbulent interface and entrainment in a boundary layer. J. Fluid Mech. 742, 119-151.

Christensen, K. T. \& AdRIAn, R. J. 2001 Statistical evidence of hairpin vortex packets in wall turbulence. J. Fluid Mech. 431, 433-443.

CSANADY, G 1973 Turbulent diffusion in the environment. Reidel, Dordrecht, The Netherlands.

Del Álamo, J. C. \& Jiménez, J. 2009 Estimation of turbulent convection velocities and corrections to Taylor's approximation. J. Fluid Mech. 640, 5-26.

Dennis, D. J. C. \& Nickels, T. B. 2008 On the limitations of Taylor's hypothesis in constructing long structures in a turbulent boundary layer. J. Fluid Mech. 614, 197-206.

Duplat, J. \& VillermauX, E. 2008 Mixing by random stirring in confined mixtures. J. Fluid Mech. 617, $51-86$.

DURBIN, P. A. 1980 A stochastic model of two-particle dispersion and concentration fluctuations in homogeneous turbulence. J. Fluid Mech. 100 (2), 279-302.

FACKRELL, J. E. 1980 A flame ionisation detector for measuring fluctuating concentration. J. Phys. E: Sci. Instrum. 13 (8), 888.

FACKRell, J. E. \& Robins, A. G. 1982 Concentration fluctuations and fluxes in plumes from point sources in a turbulent boundary layer. J. Fluid Mech. 117 (1), 26.

Finnigan, J. J., Shaw, R. H. \& Patton, E. G. 2009 Turbulence structure above a vegetation canopy. J. Fluid Mech. 637, 387-424.

GIFFORD, F. 1959 Statistical properties of a fluctuating plume dispersion model. Adv. Geophys. 6, 117-137.

HAY, J. S. \& PASQUILL, F 1959 Diffusion from a continuous source in relation to the spectrum and scale of turbulence. Adv. Geophys. 6, 345-365.

Herpin, S., Stanislas, M., Foucaut, J. M. \& Coudert, S. 2013 Influence of the Reynolds number on the vortical structures in the logarithmic region of turbulent boundary layers. J. Fluid Mech. 716, 5-50.

HuAng, J., KATUl, G. \& AlberTsOn, J. 2013 The role of coherent turbulent structures in explaining scalar dissimilarity within the canopy sublayer. Env. Fluid Mech. 13 (6), 571-599.

Hunt, J. C. R. \& WeBer, A. H. 1979 A Lagrangian statistical analysis of diffusion from a ground-level source in a turbulent boundary layer. Quart. J. Roy. Meteorol. Soc. 105 (444), 423-443.

HUtChInS, N. \& MARUSIC, I. $2007 a$ Evidence of very long meandering features in the logarithmic region of turbulent boundary layers. J. Fluid Mech. 579, 1-28.

Hutchins, N. \& MARUSIC, I. $2007 b$ Large-scale influences in near-wall turbulence. Phil. Trans. R. Soc. A 365, 647-664.

Katul, G., Kuhn, G., Schieldge, J. \& Hsieh, C. I. 1997 The ejection-sweep character of scalar fluxes in the unstable surface layer. Bound. Layer Meteorol. 83 (1), 1-26.

KLEWICKI, J. 2013 Self-similar mean dynamics in turbulent wall flows. J. Fluid Mech. 718, 596-621.

Klewicki, J. C., Murray, J. A. \& Falco, R. E. 1994 Vortical motion contributions to stress transport in turbulent boundary layers. Phys. Fluids 6 (1), 277-286.

Krishnamoorthy, L. V. \& Antonia, R. A. 1987 Temperature-dissipation measurements in a turbulent boundary layer. J. Fluid Mech. 176, 265-281.

LI, D. \& Bou-ZEID, E. 2011 Coherent structures and the dissimilarity of turbulent transport of momentum and scalars in the unstable atmospheric surface layer. Bound. Layer Meteorol. 140 (2), 243-262.

Marusic, I., McKeon, B. J., Monkewitz, P. A., Nagib, H. M., Smits, A. J. \& Sreenivasan, K. R. 
2010 Wall-bounded turbulent flows at high Reynolds numbers: Recent advances and key issues. Phys. Fluids 22 (6), 065103.

MAthis, R., Hutchins, N. \& MARUSic, I. 2009 Large-scale amplitude modulation of the small-scale structures in turbulent boundary layers. J. Fluid Mech. 628, 311-337.

МсСомв, W. 1990 The physics of fluid turbulence. Oxford University Press.

Meinhart, C. D. \& AdRIAN, R. J. 1995 On the existence of uniform momentum zones in a turbulent boundary layer. Phys. Fluids 7 (4), 694-696.

Morrill-Winter, C., Philip, J. \& Klewicki, J. 2017 An invariant representation of mean inertia: theoretical basis for a log law in turbulent boundary layers. J. Fluid Mech. 813, 594-617.

Nironi, C., Salizzoni, P., Marro, M., Mejean, P., Grosjean, N. \& Soulhac, L. 2015 Dispersion of a passive scalar fluctuating plume in a turbulent boundary layer. Part I: Velocity and concentration measurements. Bound. Layer Meteorol. 156 (3), 415-446.

Poreh, M. \& CERMAK, J. E. 1964 Study of diffusion from a line source in a turbulent boundary layer. Int. J. Heat and Mass Transfer 7 (10), 1083-1095.

Robins, A. G. 1978 Plume dispersion from ground level sources in simulated atmospheric boundary layers. Atmos. Environ. 12 (5), 1033-1044.

SAwford, B. L., Frost, C. C. \& Allan, T. C. 1985 Atmospheric boundary-layer measurements of concentration statistics from isolated and multiple sources. Bound. Layer Meteorol. 31 (3), 249-268.

DE Silva, C. M., Philip, J., Hutchins, N. \& MARUsic, I. 2017 Interfaces of uniform momentum zones in turbulent boundary layers. J. Fluid Mech. 820, 451-478.

SMIth, FB \& HAY, JS 1961 The expansion of clusters of particles in the atmosphere. Quart. J. Royal Met. Soc. 87 (371), 82-101.

Smits, A. J., McKeon, B. J. \& MARusic, I. 2011 High-Reynolds number wall turbulence. Annu. Rev. Fluid Mech. 43, 353-375.

Talluru, K. M., Hernandez-Silva, C., Philip, J. \& Chauhan, K. A. 2017a Measurements of scalar released from point sources in a turbulent boundary layer. Meas. Sci. Tech. 28 (5), 055801.

Talluru, K. M., Hernandez-Silva, C., Philip, J. \& Chauhan, K. A. $2017 b$ Measurements of velocity and concentration in a high Reynolds number turbulent boundary layer. In Tenth International Symposium on Turbulence and Shear Flow Phenomena, Chicago, USA.

Talluru, K. M., Kulandaivelu, V., Hutchins, N. \& Marusic, I. 2014 A calibration technique to correct sensor drift issues in hot-wire anemometry. Meas. Sci. Tech. 25 (10), 105304.

TAVOULARIS, S. \& CORRSIN, S. 1981 Experiments in nearly homogenous turbulent shear flow with a uniform mean temperature gradient. part 1. J. Fluid Mech. 104, 311-347.

TAYlor, G. I. 1921 Diffusion by continuous movements. Proceedings of the London Mathematical Society $2(1), 196-212$.

TENNEKes, H. \& Lumley, J. L. 1972 A first course in turbulence. MIT press.

Townsend, A. A 1958 Turbulent flow in a stably stratified atmosphere. J. Fluid Mech. 3 (4), 361-372.

Townsend, A. A. 1976 The structure of turbulent shear flow. Cambridge University Press .

VANDERWEL, C. \& TAVOUlaRIS, S. 2014 Measurements of turbulent diffusion in uniformly sheared flow. $J$. Fluid Mech. 754, 488-514.

VANDERWEL, C. \& TAVOULARIS, S. 2016 Scalar dispersion by coherent structures in uniformly sheared flow generated in a water tunnel. J. Turb. 17 (7), 633-650.

WeIL, J .C 2012 Atmospheric dispersion. In Handbook of Environmental Fluid Dynamics, Volume Two: Systems, Pollution Modeling and Measurements (ed. H. J. Fernando), pp. 163-174. CRC press.

WILLIS, G. E. \& DEARDoRfF, J. W. 1978 A laboratory study of dispersion from an elevated source within a modeled convective planetary boundary layer. Atmos. Environ. 12 (6-7), 1305-1311.

Yee, E. \& Biltoft, C. A. 2004 Concentration fluctuation measurements in a plume dispersing through a regular array of obstacles. Bound. Layer Meteorol. 111 (3), 363-415.

Yee, E., Kosteniuk, P. R., Chandler, G. M., Biltoft, C. A. \& Bowers, J. F. 1993 Statistical characteristics of concentration fluctuations in dispersing plumes in the atmospheric surface layer. Bound. Layer Meteorol. 65 (1-2), 69-109. 